\title{
Electrical bioadhesive interface for bioelectronics
}

\author{
Jue Deng ${ }^{1,2,6}$, Hyunwoo Yuk $\oplus^{2,6}$, Jingjing Wu ${ }^{1,2}$, Claudia E. Varela ${ }^{3,4}$, Xiaoyu Chen², Ellen T. Roche ${ }^{2,3}$, \\ Chuan Fei Guo $1{ }^{1 凶}$ and Xuanhe Zhao ${ }^{2,5} \llbracket$
}

\begin{abstract}
Reliable functions of bioelectronic devices require conformal, stable and conductive interfaces with biological tissues. Integrating bioelectronic devices with tissues usually relies on physical attachment or surgical suturing; however, these methods face challenges such as non-conformal contact, unstable fixation, tissue damage, and/or scar formation. Here, we report an electrical bioadhesive (e-bioadhesive) interface, based on a thin layer of a graphene nanocomposite, that can provide rapid (adhesion formation within $5 \mathrm{~s}$ ), robust (interfacial toughness $>400 \mathrm{~J} \mathrm{~m}^{-2}$ ) and on-demand detachable integration of bioelectronic devices on diverse wet dynamic tissues. The electrical conductivity $\left(>2.6 \mathrm{~S} \mathrm{~m}^{-1}\right)$ of the e-bioadhesive interface further allows bidirectional bioelectronic communications. We demonstrate biocompatibility, applicability, mechanical and electrical stability, and recording and stimulation functionalities of the e-bioadhesive interface based on ex vivo porcine and in vivo rat models. These findings offer a promising strategy to improve tissue-device integration and enhance the performance of biointegrated electronic devices.
\end{abstract}

\begin{abstract}
$\mathrm{n}$ tandem with recent advances in miniaturized and flexible electronics, the integration of bioelectronic devices in close contact with biological tissues has been intensively explored in a broad range of diagnostic and therapeutic application ${ }^{1-3}$. To ensure reliable functions of bioelectronic devices in these applications, it is crucial to establish conformal and stable contact between the device and the target tissue $e^{4,5}$. Although physical attachment of flexible bioelectronic devices on tissues has been demonstrated, such attachment is generally limited to thin devices $<5 \mu \mathrm{m}$ thick ${ }^{6-10}$. In addition, since physical attachment relies on relatively weak bonds such as van der Waals interaction ${ }^{11,12}$ or capillarity ${ }^{7}$, these thin devices may still debond from wet dynamic tissues, especially in long-term applications $^{4,13,14}$ (Fig. 1a, Extended Data Table 1 and Supplementary Fig. 1; See Supplementary Information for detailed discussion on wet tissue integration of bioelectronic devices by physical attachment).

Conventional surgical fixation through sutures has been adopted for more stable integration of bioelectronic devices, especially for devices that are not in thin-film form ${ }^{15-17}$. However, suturing can cause tissue damage and stress concentration at piercing points, and consequent scar formation ${ }^{18}$ (Fig. 1b). Furthermore, it is challenging to suture bioelectronic devices to fragile (for example, spinal cord, peripheral nerve) ${ }^{19,20}$ and dynamic (for example, heart) ${ }^{21,22}$ tissues and organs. For example, suturing of epicardial pacing-wires after heart surgery can cause tissue damage and bleeding ${ }^{23}$. More recently, bioadhesives have been explored to integrate bioelectronic devices onto tissues and organs, mostly on the $\operatorname{skin}^{24-27}$ (Supplementary Table 1). However, the poor adhesion performance (including incompatibility with wet surfaces, slow adhesion formation, weak adhesion, and difficulty in handling the adhesives) and limited electrical properties (including low electrical conductivity and high impedance) of existing bioadhesives have prevented them from providing conformal, robust yet electrically functional integration between bioelectronic devices and wet dynamic tissues ${ }^{13,27-33}$.

Here, we report an electrical bioadhesive (e-bioadhesive) interface to achieve rapid, robust, conformal and electrically conductive
\end{abstract}

integration between bioelectronic devices and various wet dynamic tissues (Fig. 1c). The e-bioadhesive interface is enabled by a thin layer of a graphene nanocomposite that can readily adhere bioelectronic devices to wet dynamic tissue surfaces. When the dry e-bioadhesive interface contacts a wet tissue surface, the e-bioadhesive interface removes water from this surface by hydration and subsequent anisotropic (only in the thickness direction) swelling, forming rapid and robust integration with the tissue surface within $5 \mathrm{~s}$ (ref. ${ }^{34}$ ) (Fig. 1d,e and Extended Data Fig. 1). After adhering on the tissue surface, the e-bioadhesive interface becomes a thin layer of a graphene nanocomposite hydrogel with high water content, softness, and stretchability, matching the mechanical properties of soft biological tissues. The e-bioadhesive interface can be made electrically conductive if it is on the electrodes of bioelectronics devices, enabling electrical recording and stimulation of the underlying tissue (Fig. 1f). Furthermore, the e-bioadhesive interface can be benignly removed from the target tissue by applying a triggering solution, allowing on-demand and atraumatic retrieval of implanted bioelectronic devices.

\section{Design and mechanism of the e-bioadhesive interface}

To implement the proposed design of the e-bioadhesive interface, we develop a graphene nanocomposite assembled on bioelectronic devices. We first introduce graphene oxide (GO) into a poly(vinyl alcohol) (PVA) hydrogel to prepare a graphene nanocomposite hydrogel that exhibits anisotropic swelling (primarily swelling orthogonal to the adhesion interface) ${ }^{35}$. The GO-PVA hydrogel is further interpenetrated with a crosslinked network of poly(acrylic acid) grafted with $N$-hydroxysuccinimide ester (PAA-NHS ester) to provide bioadhesive capability ${ }^{34}$ (Fig. $1 \mathrm{~d}$ and Extended Data Fig. 2). We then prepare the e-bioadhesive interface by drying the resultant GO-PVA-PAA-NHS ester hydrogel on the locations of the bioelectronic devices that were to adhere to tissues (Supplementary Fig. 2). The surface of the bioelectronic devices is functionalized with primary amine groups to facilitate covalent crosslinking with the e-bioadhesive interface (Fig. 1e and Extended Data Fig. 3). If the

'Department of Materials Science and Engineering, Southern University of Science and Technology, Shenzhen, China. ${ }^{2}$ Department of Mechanical Engineering, Massachusetts Institute of Technology, Cambridge, MA, USA. ${ }^{3}$ Institute for Medical Engineering and Science, Massachusetts Institute of Technology, Cambridge, MA, USA. ${ }^{4}$ Harvard-MIT Program in Health Sciences and Technology, Cambridge, MA, USA. ${ }^{5}$ Department of Civil and Environmental Engineering, Massachusetts Institute of Technology, Cambridge, MA, USA. ${ }^{6}$ These authors contributed equally: Jue Deng and Hyunwoo Yuk. ${ }^{\circ}$-mail: zhaox@mit.edu; guocf@sustech.edu.cn 
a

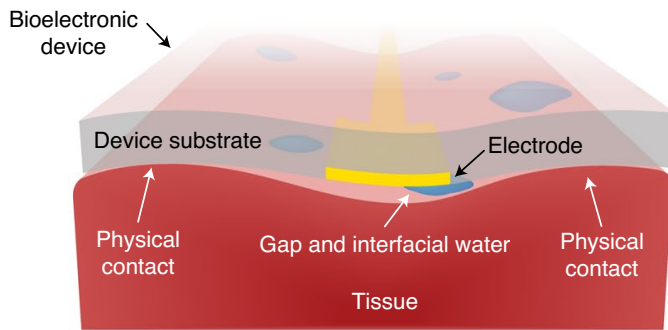

b

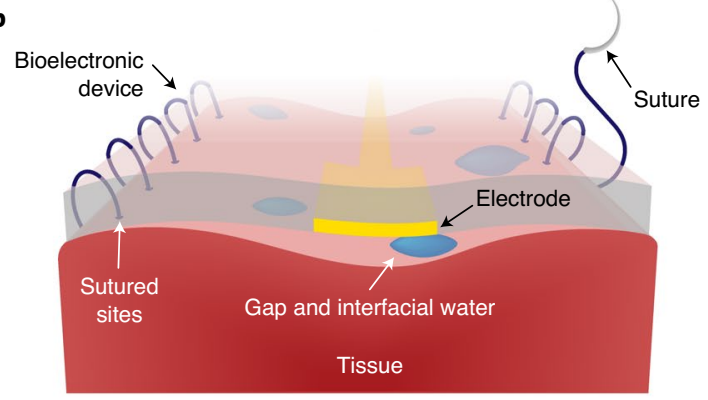

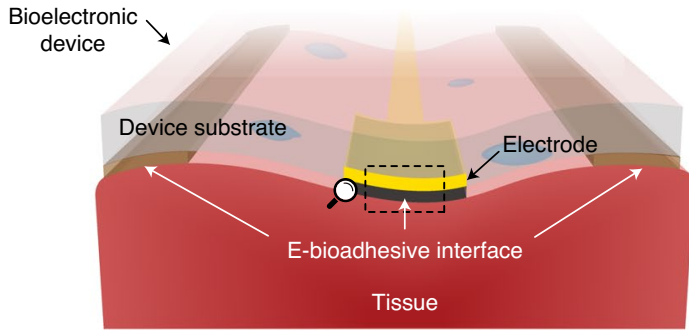

d

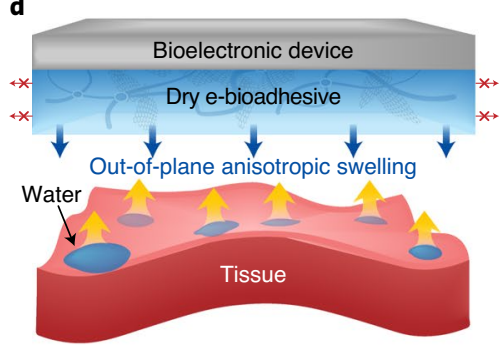

e

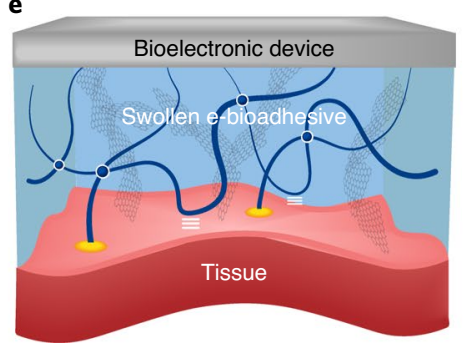

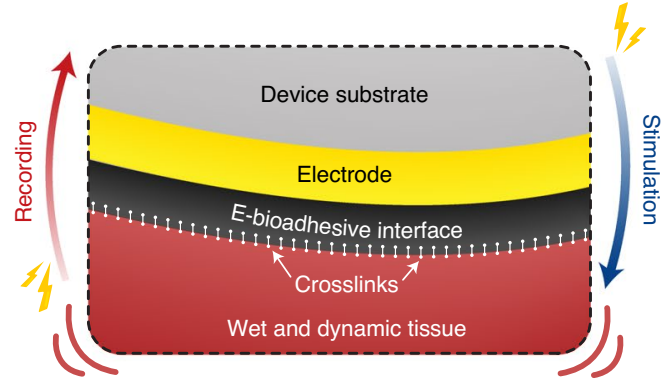

f

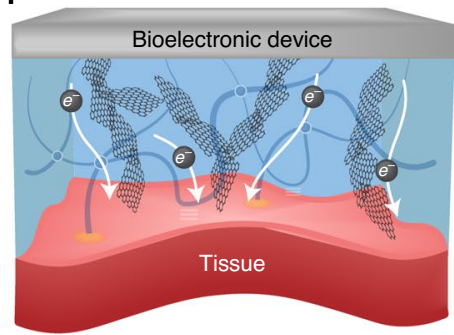

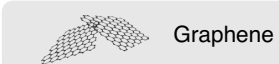

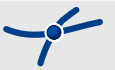

Bioadhesive polymer networks
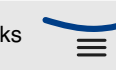

Physical crosslinks

Fig. 1 | Design and mechanism of the e-bioadhesive interface. a, Schematic illustration of the physical attachment of a bioelectronic device on a tissue. b, Schematic illustration of the suturing of a bioelectronic device on a tissue. c, Schematic illustration of a bioelectronic device adhered by the e-bioadhesive interface on a wet dynamic tissue for robust integration and bioelectronic communication (top panel); schematic illustration of a magnified cross-sectional view of the e-bioadhesive interface enabling electrical recording and stimulation between bioelectronic device and tissue (bottom panel). d-f, Materials design and properties of the graphene nanocomposite-based e-bioadhesive interface capable of providing anisotropic out-of-plane swelling (d), rapid and robust adhesion on wet tissues (e) and high electrical conductivity (f). Red arrows with crosses in $\mathbf{d}$ indicate limited in-plane swelling.

e-bioadhesive interface is placed on the electrode of the devices, the GO-PVA hydrogel can further be reduced to rGO-PVA hydrogel prior to the introduction of the bioadhesive PAA-NHS ester network. The reduction of GO results in electrical conductivity without compromising the mechanical properties because the reduction conditions adopted here cause minimal disruption of the dense hydrogen-bond-based physical crosslinks within the PVA network and between PVA and $\mathrm{GO}^{35,36}$ (Fig. If and Supplementary Fig. 3).

Once in contact with wet tissue surfaces, the carboxylic acid groups in the e-bioadhesive interface facilitate rapid absorption and removal of the interfacial water and form physical crosslinks such as hydrogen bonds and electrostatic interactions with the tissue surface under gentle pressure $(\sim 1 \mathrm{kPa})$ within $5 \mathrm{~s}$ (refs. $\left.{ }^{34,37}\right)$ (Fig. $1 \mathrm{~d}$ and Extended Data Fig. 4). The NHS ester groups in the e-bioadhesive interface can further form covalent crosslinks with primary amine groups on the tissue surface, offering long-term stable integration of bioelectronic devices in wet and dynamic physiological environments (Extended Data Fig. 4). However, the adhesion formed by the e-bioadhesive interface can be promptly reversed within $5 \mathrm{~min}$ by applying a biocompatible aqueous triggering solution $(0.5 \mathrm{M}$ sodium bicarbonate and $50 \mathrm{mM}$ L-glutathione reduced in PBS $)^{38-40}$
(Extended Data Fig. 5), allowing on-demand and atraumatic removal of the adhered bioelectronic device from the tissue.

During adhesion formation, the dry e-bioadhesive interface absorbs water and anisotropically swells orthogonally to the adhesion interface (3.6 times increase in thickness) while the length and width increase to less than 1.1 times their initial values (Fig. 2a). The e-bioadhesive interface exhibits no further dimensional change after fully swelling to its equilibrium state (Supplementary Fig. 4). The anisotropic swelling of the e-bioadhesive interface originates from the substrate-constrained drying process used in its preparation (Supplementary Fig. 2), in which the formation of rGO-PVA microstructures constrains the swelling of the e-bioadhesive along the in-plane directions (Fig. 2a) ${ }^{41}$. In comparison, substrateconstrained drying of e-bioadhesive interface without rGO leads to approximately isotropic swelling, indicating that rGO is critical for the anisotropic swelling of the e-bioadhesive interface (Fig. 2a). The anisotropic swelling property of the e-bioadhesive interface can minimize the potential geometric mismatch and subsequent distortion or delamination of the e-bioadhesive interface from bioelectronic devices in wet physiological environments. After forming adhesion on wet tissues, the swollen e-bioadhesive interface becomes a thin layer of hydrogel with tissue-like high water 

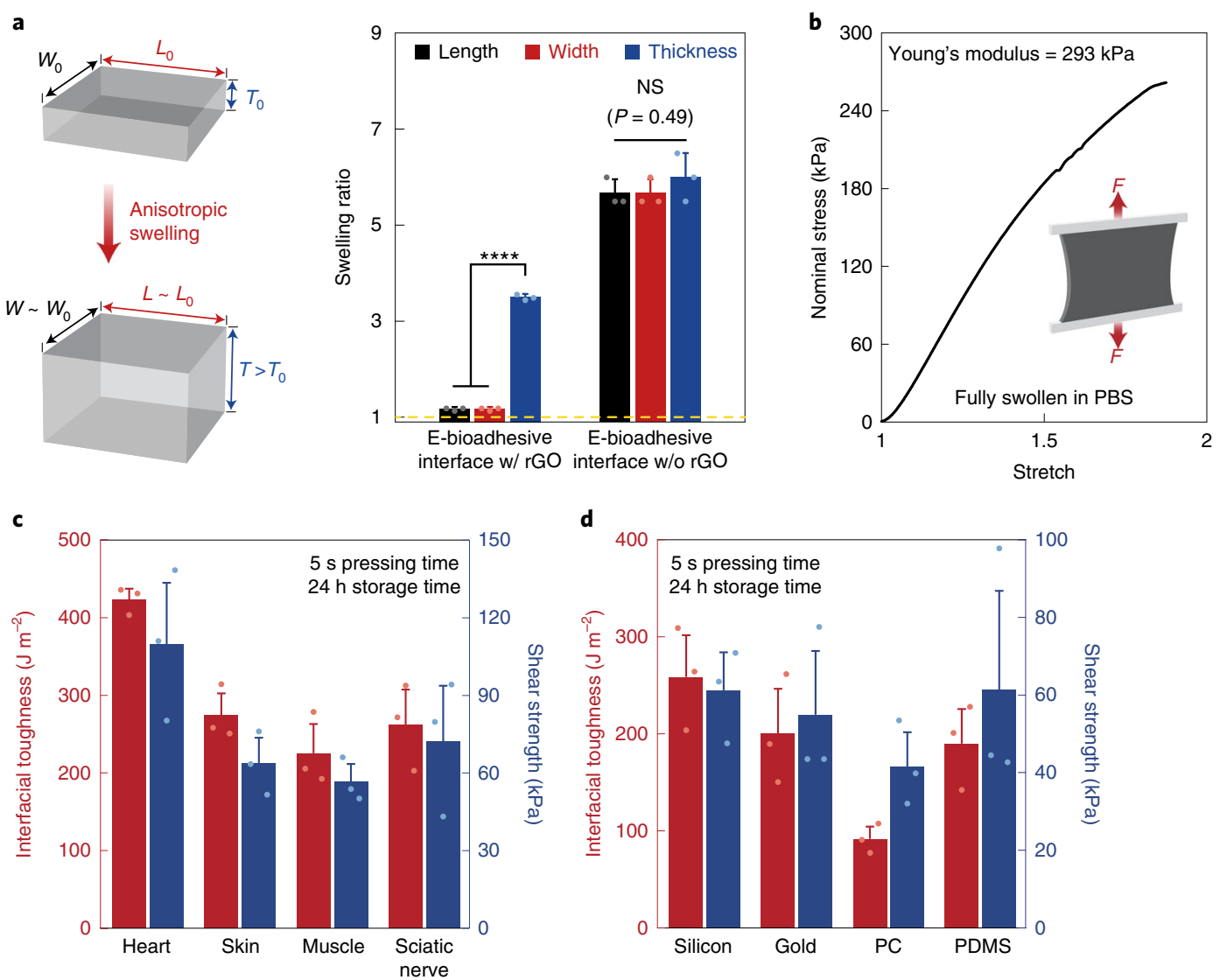

Fig. 2 | Mechanical properties of the e-bioadhesive interface. a, Schematic illustration for the anisotropic swelling (left panel) and swelling ratio in the length, width, and thickness directions of the e-bioadhesive interface with and without rGO (right panel). w/, with; w/o, without. Yellow dashed line indicates no swelling (swelling ratio equal to 1). $\mathbf{b}$, Nominal stress-stretch curve of the e-bioadhesive interface fully swollen in PBS, the slope in the curve is used to calculate the Young's modulus of fully swollen e-bioadhesive interface. F indicates tensile force applied to the sample. c, Interfacial toughness and shear strength between various tissues and polyimide substrates adhered by the e-bioadhesive interface. $\mathbf{d}$, Interfacial toughness and shear strength between porcine skin and various device materials adhered by the e-bioadhesive interface. Values in $\mathbf{a}$, $\mathbf{c}$, and $\mathbf{d}$ represent the mean and the standard deviation ( $n=3$ independent samples in $\mathbf{a}$ ). Statistical significance and $P$ values are determined by one-way ANOVA and Tukey's multiple comparison test. NS, not significant; ${ }^{\star \star \star \star} P \leq 0.0001$.

content ( $\sim 85 \%$ by volume), softness (Young's modulus of $293 \mathrm{kPa}$ ), stretchability $(>2)$, and high toughness (fracture toughness of $1,126 \mathrm{~J} \mathrm{~m}^{-2}$ ) (Fig. 2a,b and Supplementary Fig. 5). In addition, the thin thickness and the small thickness change of the e-bioadhesive interface compared with the typical dimensions of tissues, together with the tissue-like mechanical properties, can also minimize the potential compressive narrowing of underlying tissues (Extended Data Fig. 6).

\section{Adhesion performance}

To evaluate the adhesion performance of the e-bioadhesive interface, we conduct standard mechanical tests to measure the interfacial toughness $\left(180^{\circ}\right.$ peel test, ASTM F2256) and shear strength (lap-shear test, ASTM F2255) of various tissues and device materials adhered by the e-bioadhesive interface. The e-bioadhesive interface with a polyimide backing forms rapid and robust adhesion to various wet tissues with high interfacial toughness $\left(>420 \mathrm{~J} \mathrm{~m}^{-2}\right.$ for heart; $>270 \mathrm{~J} \mathrm{~m}^{-2}$ for skin; $>230 \mathrm{~J} \mathrm{~m}^{-2}$ for muscle; $>260 \mathrm{~J} \mathrm{~m}^{-2}$ for sciatic nerve) and high shear strength $(>110 \mathrm{kPa}$ for heart; $>60 \mathrm{kPa}$ for skin; $>50 \mathrm{kPa}$ for muscle; $>70 \mathrm{kPa}$ for sciatic nerve) within $5 \mathrm{~s}$ of applying gentle pressure ( $\sim 1 \mathrm{kPa}$ ) (Fig. $2 \mathrm{c}$, Supplementary Figs. 6 and $7 \mathrm{a}, \mathrm{c})$. In comparison, the interfacial toughness and shear strength of polyimide physically attached on heart are only $1.55 \mathrm{~J} \mathrm{~m}^{-2}$ and $0.21 \mathrm{kPa}$, respectively, and on skin are only $0.34 \mathrm{~J} \mathrm{~m}^{-2}$ and $0.19 \mathrm{kPa}$, respectively (Extended Data Fig. 7). The e-bioadhesive interface can also provide rapid and robust adhesion between wet tissues and various commonly used device materials with high interfacial toughness ( $>250 \mathrm{~J} \mathrm{~m}^{-2}$ for silicon; $>190 \mathrm{~J} \mathrm{~m}^{-2}$ for gold; $>200 \mathrm{~J} \mathrm{~m}^{-2}$ for polydimethylsiloxane (PDMS); $>90 \mathrm{~J} \mathrm{~m}^{-2}$ for polycarbonate (PC)) and high shear strength $(>60 \mathrm{kPa}$ for silicon; $>50 \mathrm{kPa}$ for gold; $>40 \mathrm{kPa}$ for PC; $>60 \mathrm{kPa}$ for PDMS) (Fig. $2 \mathrm{~d}$ and Supplementary Fig. 7b,d).

\section{Electrical performance}

To provide unimpeded electrical communications between the bioelectronic device and the underlying tissue, the presence of the e-bioadhesive interface on device electrodes should not substantially alter the electrical properties of the tissue-electrode interface. The e-bioadhesive interface can be readily modified to endow electrical conductivity by reducing the GO in the nanocomposite hydrogel to rGO (Fig. 3a and Supplementary Fig. 8), without compromising the favourable mechanical properties including high water content, tissue-like softness, anisotropic swelling, and rapid robust adhesion capability (Supplementary Figs. 4 and 5).

The e-bioadhesive interface exhibits a stable electrical conductivity of $>2.6 \mathrm{~S} \mathrm{~m}^{-1}$ during a 14 day incubation in $\mathrm{PBS}$ at $37^{\circ} \mathrm{C}$, both with and without endogenous enzymes (for example, collagenase). The conductivity of the e-bioadhesive interface is substantially 

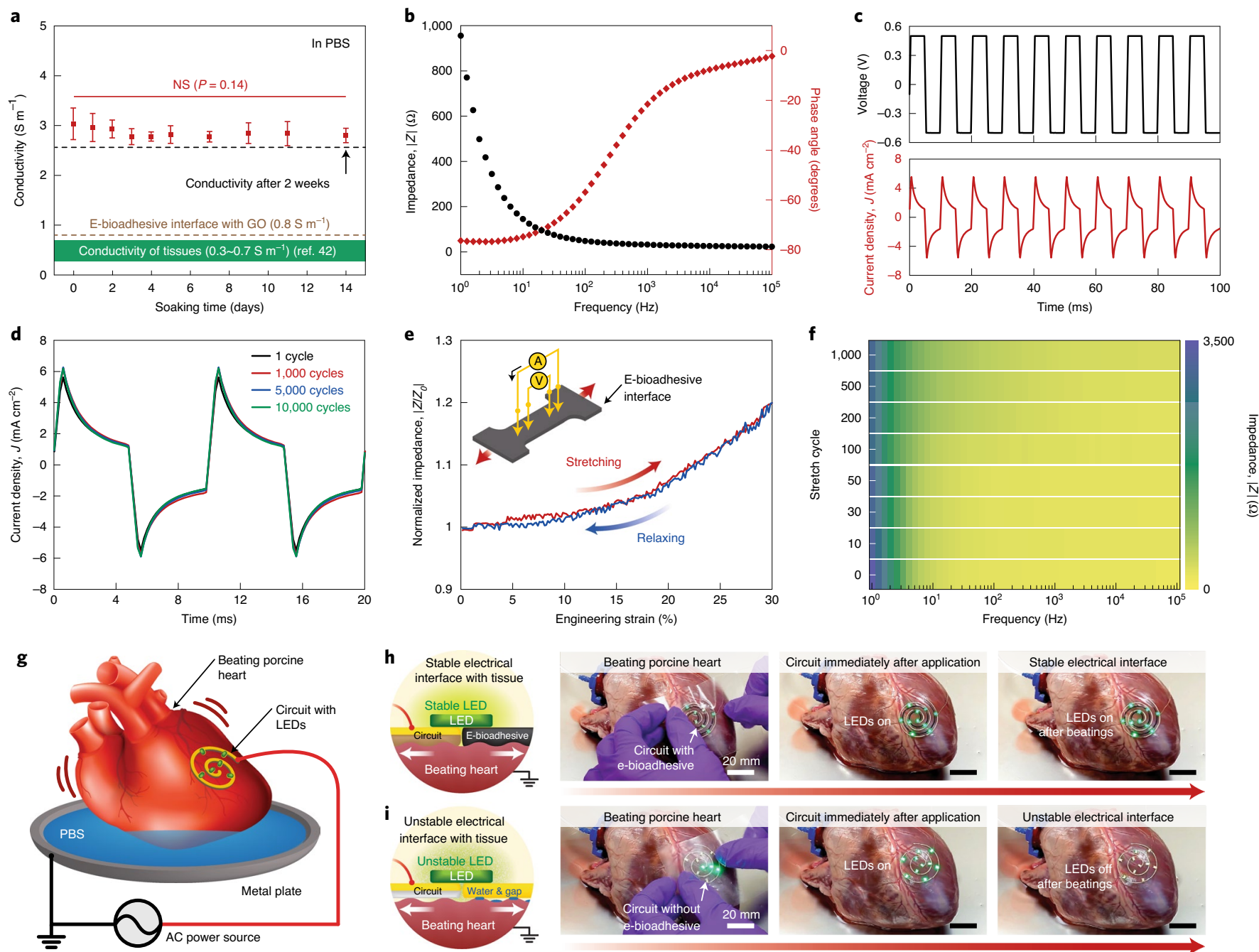

Fig. 3 | Electrical properties of the e-bioadhesive interface. a, Conductivity of the e-bioadhesive interface in PBS over 14 days at $37^{\circ} \mathrm{C}$. Values represent the mean and the standard deviation ( $n=8$ independent samples). Statistical significance and $P$ values are determined by one-way ANOVA and Tukey's multiple comparison test. NS, not significant. b. Electrical impedance spectroscopy measurements in the thickness direction of the e-bioadhesive interface fully swollen in PBS. c, Charge injection curves of the e-bioadhesive interface with biphasic pulses of $10 \mathrm{~ms}$ and $\pm 0.5 \mathrm{~V}$ versus $\mathrm{Ag} / \mathrm{AgCl}$. d, $\mathrm{Charge}$ injection curves of the e-bioadhesive interface at 1, 1,000,5,000 and 10,000 cycles. e, Impedance in the thickness direction of the e-bioadhesive interface at $1 \mathrm{kHz}$ during cyclic tensile deformations measured by ammeter $(A)$ and voltmeter $(V)$. $\mathbf{f}$, Impedance in the thickness direction of the e-bioadhesive interface for $1-10,000 \mathrm{~Hz}$ at different stretch cycles $(1,10,30,50,100,300,500$ and 1,000). $\mathbf{g}$. Schematic illustration of a circuit with LEDs adhered on an ex vivo beating porcine heart. $\mathbf{h}, \mathbf{i}$, Schematic illustrations and images of a circuit with LEDs with $(\mathbf{h})$ and without (i) the e-bioadhesive interface adhered on an ex vivo porcine heart.

higher than that of biological tissues $\left(0.3-0.7 \mathrm{~S} \mathrm{~m}^{-1}\right)^{42}$ (Fig. 3a and Supplementary Fig. 9). Moreover, the thin thickness $(<100 \mu \mathrm{m})$ and the conformal adhesion of the e-bioadhesive interface can further reduce interconnection and contact resistance, respectively. As a result, the e-bioadhesive interface shows a low impedance $(<50 \Omega)$ at physiologically relevant frequencies of $10^{2}-10^{5} \mathrm{~Hz}$ (ref. ${ }^{5}$ ) (Fig. $3 \mathrm{~b}$ ), minimizing the unfavourable increase of electrical impedance at the device-tissue interface. We further characterize the charge injection capability (CIC) of the e-bioadhesive interface to evaluate its effect on the electrical stimulation efficacy of bioelectronic devices (Fig. 3c). The e-bioadhesive interface shows a CIC value of $\sim 25 \mu \mathrm{Ccm}^{-2}$, comparable to that of metallic electrodes $\left(10-50 \mu \mathrm{C} \mathrm{cm}^{-2}\right)^{43}$, indicating that the presence of the e-bioadhesive interface does not impair the electrical stimulation efficacy (Fig. 3d). We further evaluate the electrochemical stability of the e-bioadhesive interface by cyclic CIC measurements. The e-bioadhesive interface exhibits a $<5 \%$ decrease in CIC value after 10,000 charging and discharging cycles (Fig. 3d). Moreover, the out-of-plane impedance of the e-bioadhesive interface exhibits a negligible hysteresis (Fig. 3e) and fluctuation (Fig. 3f) during cyclic tensile deformations at a physiologically relevant strain level (for example, a 30\% tensile engineering strain), further supporting the stability of the e-bioadhesive interface in dynamic physiological conditions.

To demonstrate the robust mechanical integration and stable electrical communication between bioelectronic devices and wet dynamic tissues through the e-bioadhesive interface, we apply a circuit with light-emitting diodes (LEDs) with and without the e-bioadhesive interface onto a beating ex vivo porcine heart (by introducing cyclical, pressurized air inputs into the heart chambers to mimic heartbeats, Fig. $3 g$ ). The circuit with the e-bioadhesive interface can form rapid and robust integration as well as stable electrical communication with the porcine heart (Fig. $3 \mathrm{~h}$ and 


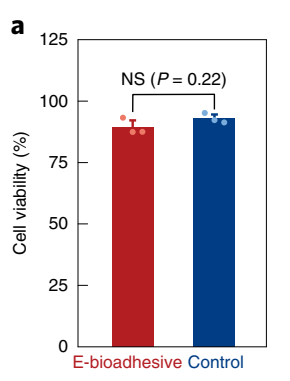

b

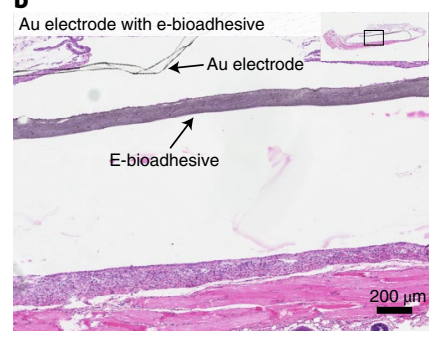

c

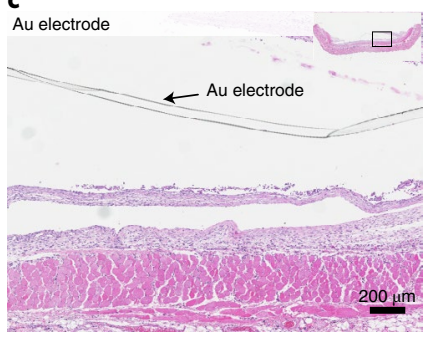

d

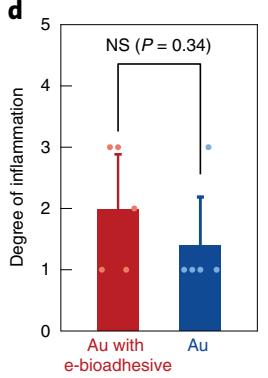

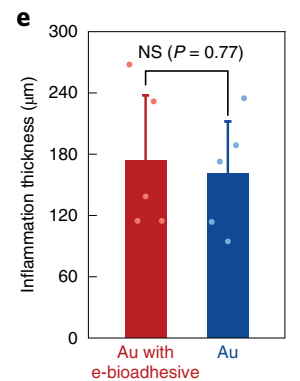

f
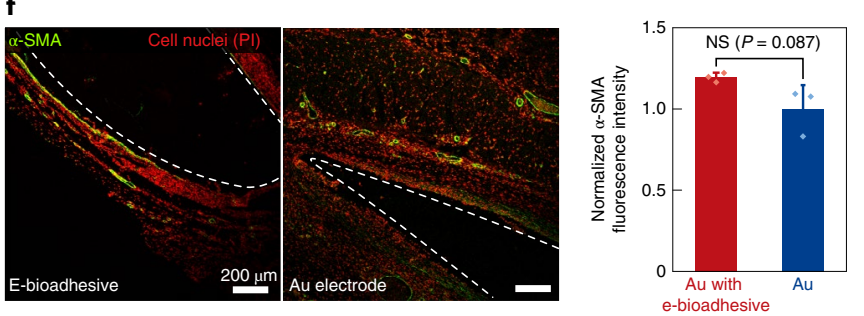

g
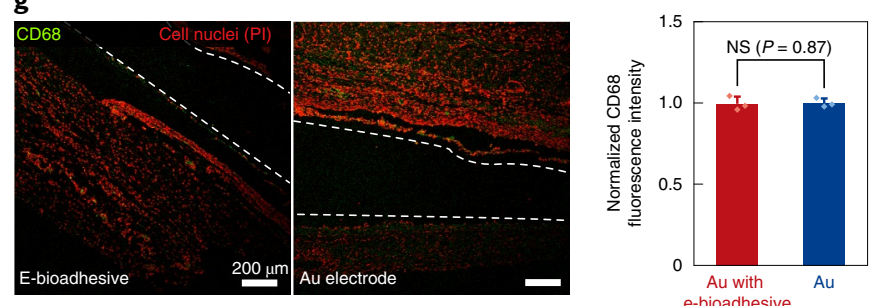

i
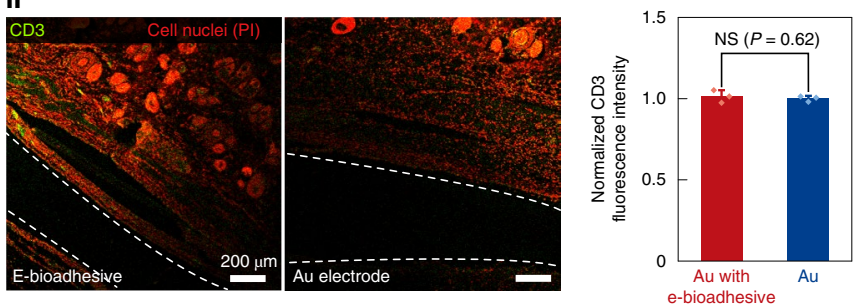

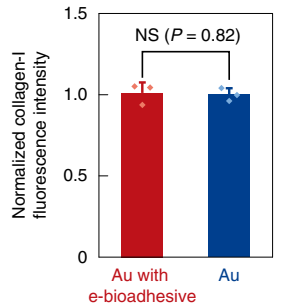

Fig. 4 | In vitro and in vivo biocompatibility of the e-bioadhesive interface. a, In vitro cell viability of rat cardiomyocytes based on LIVE/DEAD assay after $24 \mathrm{~h}$ culture in a control media (DMEM) and in an e-bioadhesive-incubated media. b,c, Representative histology images stained with haematoxylin and eosin for gold electrodes with (b) and without (c) the e-bioadhesive interface after rat subcutaneous implantation for 14 days. Five independent experiments were conducted with similar results. d,e, Degree of tissue inflammation (d) and inflammation thickness (e) when using gold electrodes with and without the e-bioadhesive interface evaluated by a blinded pathologist ( 0 , normal; 1 , very mild; 2 , mild; 3 , moderate; 4 , severe; 5 , very severe).

$\mathbf{f}-\mathbf{i}$, Representative immunofluorescence images and normalized fluorescence intensity obtained with gold electrodes with and without the e-bioadhesive interface after subcutaneous implantation in rat for 14 days. Cell nuclei are stained with propidium iodide (PI, red). Green fluorescence corresponds to the expression of fibroblasts ( $\alpha$-SMA, $\mathbf{f}$ ), macrophages (CD68, $\mathbf{g}), T$ cells $(C D 3, \mathbf{h})$, and collagen (collagen-I, $\mathbf{i})$, respectively. Values in $\mathbf{a}$ and $\mathbf{d}-\mathbf{i}$ represent the mean and the standard deviation ( $n=3$ independent samples in $\mathbf{a}$ and $\mathbf{f}-\mathbf{i}$, and $n=5$ independent samples in $\mathbf{d}$ and $\mathbf{e}$ ). Statistical significance and $P$ values are determined by two-sided Student's $t$-test. NS, not significant.

Supplementary Video 1). In contrast, the circuit without the e-bioadhesive interface exhibits unstable adhesion with the beating heart, resulting in the LEDs blinking along with the beating of the heart (Fig. 3i and Supplementary Video 2).

\section{Biocompatibility}

To evaluate the biocompatibility of the e-bioadhesive interface, we perform a set of in vitro and in vivo characterizations based on rat models (Fig. 4). A cell culture media (Dulbecco's modified eagle medium, DMEM) conditioned with the e-bioadhesive interface shows comparable in vitro cytotoxicity of rat cardiomyocytes to a control (pristine DMEM) after $24 \mathrm{~h}$ culture (Fig. $4 \mathrm{a}$ and Supplementary Fig. 10). We further evaluate the in vivo biocompatibility of the e-bioadhesive interface based on dorsal subcutaneous implantation in a rat model for 14 days (Fig. 4b,c). Histological assessments by a blinded pathologist demonstrate that both gold electrodes with and without the e-bioadhesive interface show comparable tissue responses without severe inflammatory response 14 days after implantation $(P=0.34$, Fig. 4 d). Furthermore, there is no significant difference $(P=0.77)$ in the thickness of inflammation between the gold electrodes with and without the e-bioadhesive interface (Fig. 4e).

To further investigate the in vivo biocompatibility of the e-bioadhesive interface, we perform immunofluorescence staining of various markers for fibroblasts ( $\alpha$-smooth muscle actin ( $\alpha$-SMA),
Fig. 4f), macrophages (CD68, Fig. 4g), T cells (CD3, Fig. 4h), and collagen (collagen-I, Fig. 4i) related to inflammatory and foreign body responses. Based on the immunofluorescence analysis, the gold electrodes with and without the e-bioadhesive interface exhibited no significant difference in the expression of $\alpha$-SMA $(P=0.087)$, CD68 $(P=0.87)$, CD3 $(P=0.62)$, and collagen-I $(P=0.82)$, indicating that the in vivo biocompatibility of the e-bioadhesive interface is comparable to that of conventional gold electrodes. The immunohistochemistry staining of the same markers further confirmed that the presence of the e-bioadhesive interface on gold electrodes does not generate any significant difference in terms of the expression of $\alpha$-SMA, CD68, CD3, and collagen-I (Extended Data Fig. 8).

We further quantitatively evaluate the in vivo stability of the mechanical and electrical properties of the e-bioadhesive interface by measuring the conductivity, impedance, and interfacial toughness of two gold electrodes adhered by the e-bioadhesive interface 14 days after implantation in rat dorsal subcutaneous pockets (Extended Data Fig. 9). We find that the conductivity (Extended Data Fig. 9a), impedance (Extended Data Fig. 9b), and interfacial toughness (Extended Data Fig. 9c) of the in vivo implanted samples after 14 days were not statistically significantly different $(P>0.05)$ compared with the samples incubated in PBS for 1 and 14 days, demonstrating the in vivo stability of the e-bioadhesive interface (Fig. 5). 
a

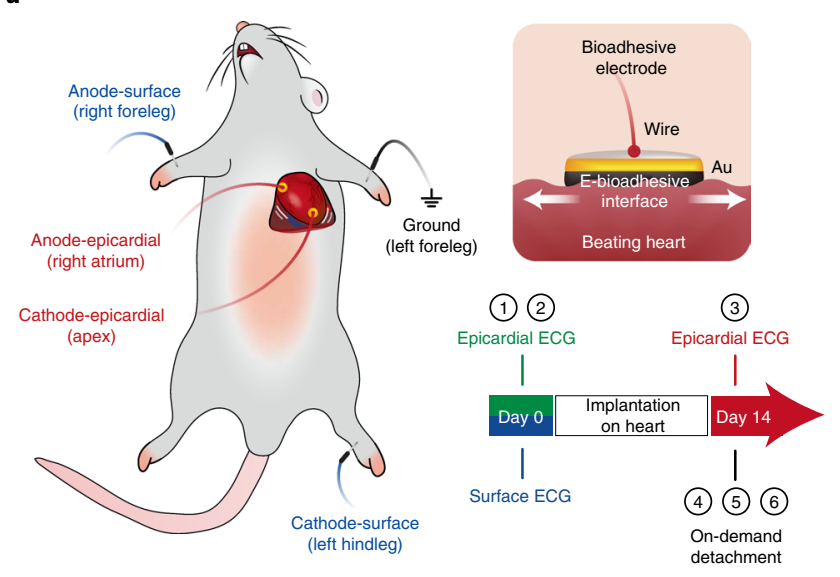

b

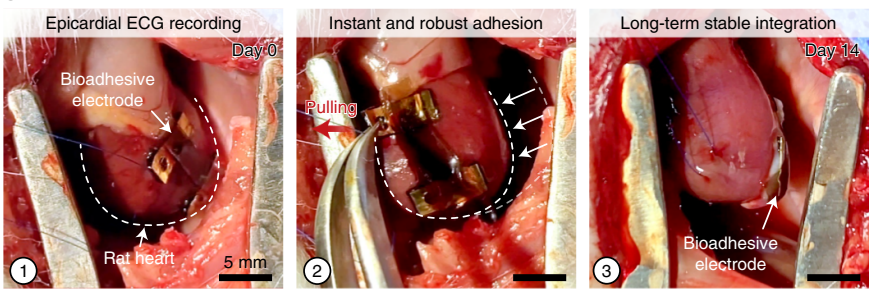

Triggering solution application

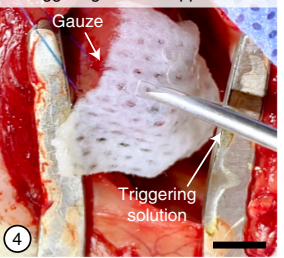

On-demand removal of bioadhesive electrode

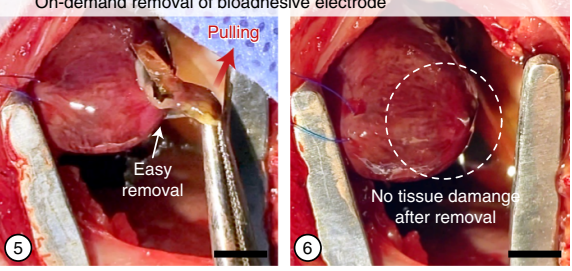

c

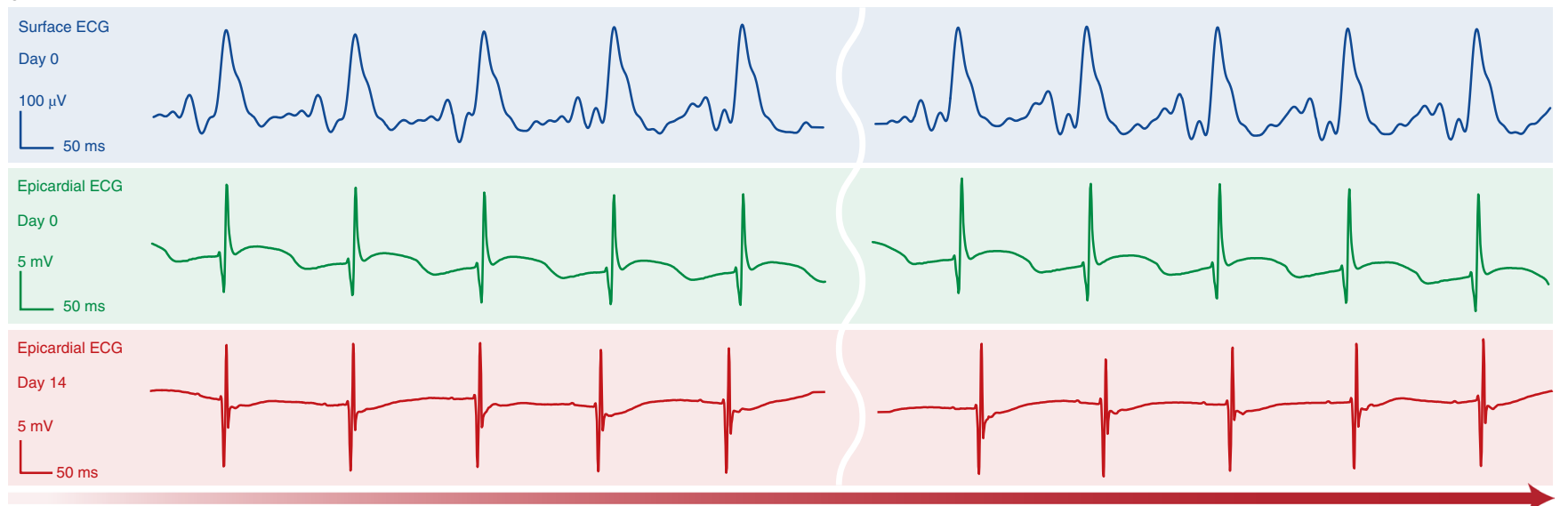

d
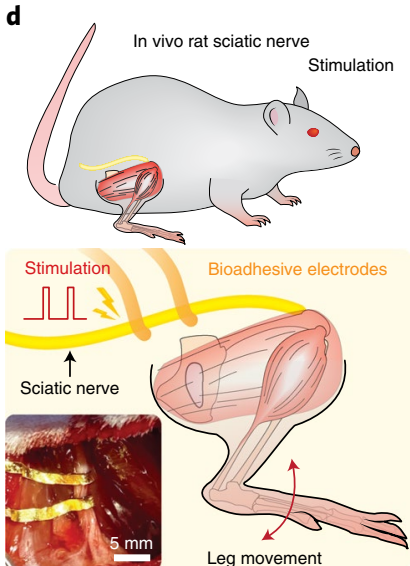

e
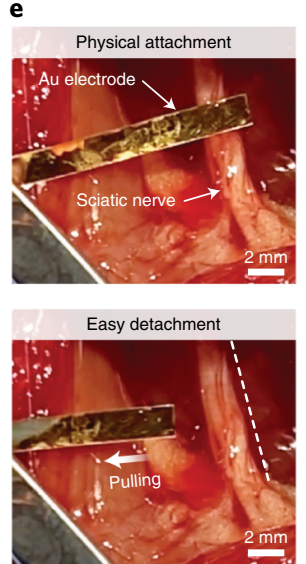

f
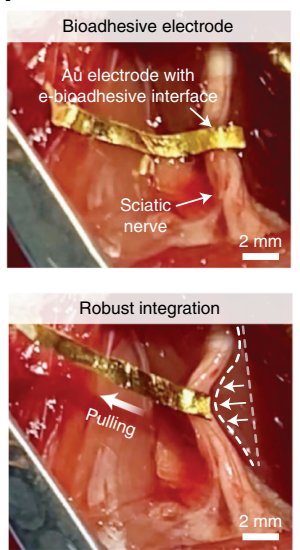

g
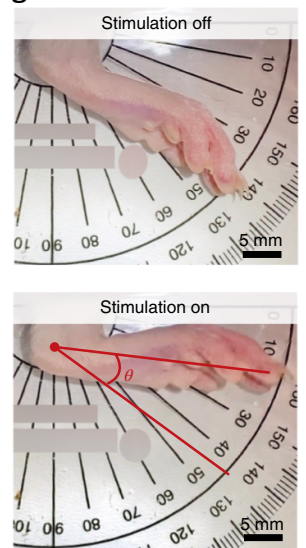

h

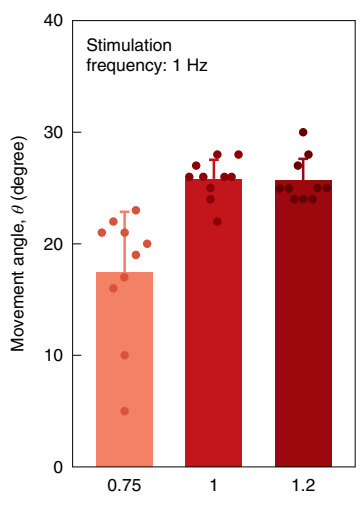

Stimulation voltage (V)

Fig. 5 | In vivo recording and stimulation via the e-bioadhesive interface. a, Schematic illustrations of an in vivo surface and epicardial ECG recording setup and study design. b, Representative images of in vivo epicardial ECG recordings of a rat heart on day 0 and day 14 of in vivo implantation and on-demand detachment of the bioadhesive electrode on day 14 after implantation. c, Representative surface ECG signals recorded by conventional needle electrodes and the epicardial ECG signals recorded by the bioadhesive electrode on day 0 and day 14 of in vivo implantation. $\mathbf{d}$, Schematic illustrations and an image of the sciatic nerve stimulation setup. $\mathbf{e}$, Physical attachment of a stimulation electrode on a sciatic nerve without the e-bioadhesive interface. $\mathbf{f}$, Robust integration of a stimulation electrode on a sciatic nerve with the e-bioadhesive interface. $\mathbf{g}$, Images of the ankle joint movement in response to electrical stimulation via the e-bioadhesive interface. $\mathbf{h}$, Movement angle of the ankle joint in response to electrical stimulation via the e-bioadhesive interface. Values represent the mean of movement angle and the standard deviation ( $n=10$ independent measurements from different animals).

\section{Bioelectronic functionalities}

To evaluate the in vivo bioelectronic functionality of the e-bioadhesive interface, we record an in situ epicardial electrocardiogram (ECG, Fig. 5a-c) and electrically stimulated a sciatic nerve (Fig. $5 \mathrm{~d}-\mathrm{h}$ ) by gold electrodes with the e-bioadhesive interface based on a rat model. To record the epicardial ECG, anodic and cathodic bioadhesive electrodes (gold electrodes with the e-bioadhesive interface) are applied to the right atrium and apex of the rat heart, respectively (Fig. 5a). Simultaneously, we also record a surface ECG by using conventional needle electrodes to evaluate the quality of 
the epicardial ECG and to monitor possible arrhythmia generated by the bioadhesive electrodes. The rapid and robust adhesion of the e-bioadhesive interface enables the electrodes to firmly adhere to the surface of a beating rat heart within a few seconds upon contact and gentle pressing. The robust integration is further validated by gently pulling the adhered electrode and subsequently lifting the whole heart by this electrode (Fig. 5b).

The e-bioadhesive interface allows stable recording of the epicardial ECG without an observable baseline shift or high-amplitude noise from the beating rat heart once the bioadhesive electrodes have adhered to the epicardial tissues (Fig. 5b,c and Supplementary Video 2). Notably, the surface ECG does not show any observable sign of arrhythmia caused by the bioadhesive electrodes, demonstrating the compatibility of the e-bioadhesive interface with electrically active tissues and organs such as the heart. After 14 days of in vivo implantation, the bioadhesive electrodes maintain a robust integration with the rat heart and allowed recording of epicardial ECG signals with waveforms and signal-to-noise ratios comparable to the epicardial ECG signals recorded immediately after the implantation of the bioadhesive electrodes on day 0 (Fig. 5b,c), validating the in vivo stability and efficacy of the e-bioadhesive interface. Taking advantage of the triggerable detachment capability of the e-bioadhesive interface ${ }^{40}$, we further demonstrat on-demand atraumatic removal of the adhered bioadhesive electrodes after 14 days of in vivo implantation (Fig. 5b and Supplementary Fig. 11).

For in vivo electrical stimulation of the sciatic nerve, we use flexible electrodes (50- $\mu$ m-thick gold-coated polyimide) with the e-bioadhesive interface (Fig. 5d). The electrodes without the e-bioadhesive interface are easily detached from the sciatic nerve with a gentle pull (Fig. 5e). In contrast, the electrodes with the e-bioadhesive interface are robustly integrated with the sciatic nerve, and maintain conformal and robust integration under gentle pulling (Fig. 5f). Under electrical stimulations $(0.75-1.2 \mathrm{~V}$ at $1 \mathrm{~Hz}$, biphasic charge balanced rectangular voltage pulses $)^{16,44,45}$, periodic and stable ankle joint movements $\left(>25^{\circ}\right)$ are observed (Fig. $5 \mathrm{~g}$,h and Supplementary Video 3), demonstrating the stable electrical stimulation of the sciatic nerve via the e-bioadhesive interface.

\section{Outlook}

We have introduced and demonstrated an e-bioadhesive interface to achieve rapid, robust, atraumatic and electrically functional integration of bioelectronic devices to diverse wet dynamic tissues. Together with the broad applicability of the e-bioadhesive interface to a wide range of materials and surface topologies, the systematically validated in vivo performance of the e-bioadhesive interface can facilitate ready adoption and conversion of existing bioelectronic devices for various applications. The e-bioadhesive interface not only offers a promising solution to addressing the long-standing challenges in tissue-device integration but also provides valuable insights for future development of biointegrative electronics with improved functionalities and efficacy in wet physiological environments.

\section{Online content}

Any methods, additional references, Nature Research reporting summaries, source data, extended data, supplementary information, acknowledgements, peer review information; details of author contributions and competing interests; and statements of data and code availability are available at https://doi.org/10.1038/ s41563-020-00814-2.

Received: 18 January 2020; Accepted: 26 August 2020;

Published online: 28 September 2020

\section{References}

1. Rogers, J. A., Someya, T. \& Huang, Y. Materials and mechanics for stretchable electronics. Science 327, 1603-1607 (2010)
2. Someya, T., Bao, Z. \& Malliaras, G. G. The rise of plastic bioelectronics. Nature 540, 379-385 (2016).

3. Feiner, R. \& Dvir, T. Tissue-electronics interfaces: from implantable devices to engineered tissues. Nat. Rev. Mater. 3, 17076 (2018).

4. Schiavone, G. \& Lacour, S. P. Conformable bioelectronic interfaces: mapping the road ahead. Sci. Transl. Med. 11, eaaw5858 (2019).

5. Yuk, H., Lu, B. \& Zhao, X. Hydrogel bioelectronics. Chem. Soc. Rev. 48, 1642-1667 (2019).

6. Zhang, Y. et al. Climbing-inspired twining electrodes using shape memory for peripheral nerve stimulation and recording. Sci. $A d v . \mathbf{5}$, eaaw1066 (2019).

7. Kim, D.-H. et al. Dissolvable films of silk fibroin for ultrathin conformal bio-integrated electronics. Nat. Mater. 9, 511-517 (2010).

8. Park, J. et al. Electromechanical cardioplasty using a wrapped elasto-conductive epicardial mesh. Sci. Transl. Med. 8, 344 ra386 (2016).

9. Jeong, J. W. et al. Materials and optimized designs for human-machine interfaces via epidermal electronics. Adv. Mater. 25, 6839-6846 (2013).

10. Sim, K. et al. Metal oxide semiconductor nanomembrane-based soft unnoticeable multifunctional electronics for wearable human-machine interfaces. Sci. Adv. 5, eaav9653 (2019).

11. Kim, D.-H. et al. Epidermal electronics. Science 333, 838-843 (2011).

12. Kaltenbrunner, M. et al. An ultra-lightweight design for imperceptible plastic electronics. Nature 499, 458 (2013).

13. Lee, S. et al. A strain-absorbing design for tissue-machine interfaces using a tunable adhesive gel. Nat. Commun. 5, 5898 (2014).

14. Ouyang, H. et al. Symbiotic cardiac pacemaker. Nat. Commun. 10, 1821 (2019).

15. Boutry, C. M. et al. Biodegradable and flexible arterial-pulse sensor for the wireless monitoring of blood flow. Nat. Biomed. Eng. 3, 47-57 (2019).

16. Koo, J. et al. Wireless bioresorbable electronic system enables sustained nonpharmacological neuroregenerative therapy. Nat. Med. 24, 1830-1836 (2018).

17. Boutry, C. M. et al. A stretchable and biodegradable strain and pressure sensor for orthopaedic application. Nat. Electron. 1, 314-321 (2018).

18. Chang, E. I. et al. Vascular anastomosis using controlled phase transitions in poloxamer gels. Nat. Med. 17, 1147-1152 (2011).

19. Minev, I. R. et al. Electronic dura mater for long-term multimodal neural interfaces. Science 347, 159-163 (2015).

20. Formento, E. et al. Electrical spinal cord stimulation must preserve proprioception to enable locomotion in humans with spinal cord injury. Nat. Neurosci. 21, 1728-1741 (2018).

21. Roche, E. T. et al. Soft robotic sleeve supports heart function. Sci. Transl. Med. 9, eaaf3925 (2017).

22. Whyte, W. et al. Sustained release of targeted cardiac therapy with a replenishable implanted epicardial reservoir. Nat. Biomed. Eng. 2, 416-428 (2018)

23. Nido, P. D. \& Goldman, B. S. Temporary epicardial pacing after open heart surgery: complications and prevention. J. Card. Surg. 4, 99-103 (1989).

24. Yang, S. et al. "Cut-and-paste" manufacture of multiparametric epidermal sensor systems. Adv. Mater. 27, 6423-6430 (2015).

25. Koh, A. et al. A soft, wearable microfluidic device for the capture, storage, and colorimetric sensing of sweat. Sci. Transl. Med. 8, 366ral65 (2016).

26. Liao, M. et al. Wearable, healable, and adhesive epidermal sensors assembled from mussel-inspired conductive hybrid hydrogel framework. Adv. Funct. Mater. 27, 1703852 (2017).

27. Liang, S. et al. Paintable and rapidly bondable conductive hydrogels as therapeutic cardiac patches. Adv. Mater. 30, 1704235 (2018).

28. Sekitani, T. et al. Ultraflexible organic amplifier with biocompatible gel electrodes. Nat. Commun. 7, 11425 (2016).

29. Yamagishi, K. et al. Tissue-adhesive wirelessly powered optoelectronic device for metronomic photodynamic cancer therapy. Nat. Biomed. Eng. 3, 27-36 (2019).

30. Keplinger, C. et al. Stretchable, transparent, ionic conductors. Science 341, 984-987 (2013).

31. Li, J. et al. Tough adhesives for diverse wet surfaces. Science 357, 378-381 (2017).

32. Rose, S. et al. Nanoparticle solutions as adhesives for gels and biological tissues. Nature 505, 382-385 (2014)

33. Liu, J. et al. Intrinsically stretchable electrode array enabled in vivo electrophysiological mapping of atrial fibrillation at cellular resolution. Proc. Natl Acad. Sci. USA 117, 14769-14778 (2020).

34. Yuk, H. et al. Dry double-sided tape for adhesion of wet tissues and devices. Nature 575, 169-174 (2019).

35. Yang, J.-H. \& Lee, Y.-D. Highly electrically conductive rGO/PVA composites with a network dispersive nanostructure. J. Mater. Chem. 22, 8512-8517 (2012).

36. Liu, J. et al. Fatigue-resistant adhesion of hydrogels. Nat. Commun. 11, 1-9 (2020).

37. Mao, X., Yuk, H. \& Zhao, X. Hydration and swelling of dry polymers for wet adhesion. J. Mech. Phys. Solids 137, 103863 (2020). 
38. Liu, J. et al. Triggerable tough hydrogels for gastric resident dosage forms. Nat. Commun. 8, 124 (2017).

39. Li, W. et al. Tough bonding, on-demand debonding, and facile rebonding between hydrogels and diverse metal surfaces. Adv. Mater. 31, 1904732 (2019).

40. Chen, X., Yuk, H., Wu, J., Nabzdyk, C. S. \& Zhao, X. Instant tough bioadhesive with triggerable benign detachment. Proc. Natl Acad. Sci. USA 117, 15497-15503 (2020).

41. Kashyap, S., Pratihar, S. K. \& Behera, S. K. Strong and ductile graphene oxide reinforced PVA nanocomposites. J. Alloy. Compd. 684, 254-260 (2016).

42. Gabriel, C., Peyman, A. \& Grant, E. H. Electrical conductivity of tissue at frequencies below 1 MHz. Phys. Med. Biol. 54, 4863 (2009).
43. Cogan, S. F. Neural stimulation and recording electrodes. Annu. Rev. Biomed. Eng. 10, 275-309 (2008).

44. Liu, Y. et al. Soft and elastic hydrogel-based microelectronics for localized low-voltage neuromodulation. Nat. Biomed. Eng. 3, 58-68 (2019).

45. Zheng, H. et al. A shape-memory and spiral light-emitting device for precise multisite stimulation of nerve bundles. Nat. Commun. 10, 2790 (2019).

Publisher's note Springer Nature remains neutral with regard to jurisdictional claims in published maps and institutional affiliations.

(C) The Author(s), under exclusive licence to Springer Nature Limited 2020 


\section{Methods}

Preparation of the e-bioadhesive interface. PVA solution $(20 \% \mathrm{w} / \mathrm{w}, 99+\%$ hydrolysed $\left.M_{\mathrm{w}} 89,000-98,000\right)$ and GO solution (Graphene Supermarket) were mixed in a volume ratio of $1: 1$ and stirred for $48 \mathrm{~h}$ to obtain a homogeneous GO-PVA solution. The GO-PVA solution was poured into a glass mould, followed by freezing at $-20^{\circ} \mathrm{C}$ for $8 \mathrm{~h}$ and thawing at $25^{\circ} \mathrm{C}$ for $3 \mathrm{~h}$ to form a GO-PVA hydrogel. The GO-PVA hydrogel was further dried in an incubator at $37^{\circ} \mathrm{C}$ for $1 \mathrm{~h}$ and annealed at $100^{\circ} \mathrm{C}$ for $1 \mathrm{~h}$ to obtain a dry GO-PVA film $(23.7 \% \mathrm{w} / \mathrm{w} \mathrm{GO})$, which was used for preparation of the non-electrically conductive e-bioadhesive interface. For preparation of the electrically conductive e-bioadhesive interface, the dry-annealed GO-PVA film was further reduced into rGO-PVA by immersing in $\mathrm{Na}_{2} \mathrm{~S}_{2} \mathrm{O}_{4}(0.15 \mathrm{M})$ and $\mathrm{NaOH}(0.5 \mathrm{M})$ solution at $65^{\circ} \mathrm{C}$ for $1 \mathrm{~h}$, followed by rinsing and immersing in deionized water for $4 \mathrm{~h}$ three times to thoroughly remove the residual $\mathrm{Na}_{2} \mathrm{~S}_{2} \mathrm{O}_{4}$ and $\mathrm{NaOH}$. Unless otherwise indicated, the electrically conductive version of the e-bioadhesive interface (based on rGO-PVA) was used (Figs. 2-5).

To introduce the bioadhesive PAA-NHS ester network, the GO-PVA or rGO-PVA film was immersed in an aqueous acrylic acid solution $(30 \% \mathrm{w} / \mathrm{w}$ acrylic acid, $0.03 \% \mathrm{w} / \mathrm{w} N, N^{\prime}$-bis(acryloyl)cystamine and $0.15 \% \mathrm{w} / \mathrm{w} 2,2^{\prime}$-azobis $(2-\mathrm{m}$ ethylpropionamidine) dihydrochloride in deionized water) for $2 \mathrm{~h}$. The soaked hydrogel was sealed and heated at $70^{\circ} \mathrm{C}$ for $30 \mathrm{~min}$ to form the PAA network, followed by drying on a glass substrate (to ensure constrained drying) under nitrogen flow. To introduce NHS ester groups into the PAA network, the dry film was immersed in an aqueous solution of 1-ethyl-3-(-3-dimethylaminopropyl) carbodiimide $(0.5 \% \mathrm{w} / \mathrm{w})$ and $N$-hydroxysulfosuccinimide sodium salt $(0.25 \% \mathrm{w} / \mathrm{w})$ for $5 \mathrm{~min}$ in room temperature, providing the as-prepared rGO-PVA-PAA-NHS hydrogel. This hydrogel was then further dried on a glass substrate or a device under nitrogen flow to prepare the dry e-bioadhesive interface (Supplementary Fig. 2). The dry samples were sealed in a plastic bag with dessicant and stored at $-20^{\circ} \mathrm{C}$ before use.

To prepare the e-bioadhesive interface without GO, we used PVA hydrogel without GO but otherwise followed the same process.

Surface functionalization of device materials. To produce covalent coupling between bioelectronic devices and the e-bioadhesive interface, various device materials were surface functionalized with primary amine groups (Extended Data Fig. 3). The substrates were first activated or cleaned by oxygen plasma treatment applied for $3 \mathrm{~min}$ ( $30 \mathrm{~W}$ power, Harrick Plasma) followed by incubation in the functionalization solution. For surface functionalization of glass and PDMS, (3-aminopropyl)triethoxysilane solution ( $1 \% \mathrm{w} / \mathrm{w}$ in $50 \%$ ethanol in deionized water) was used with an incubation time of $3 \mathrm{~h}$ at room temperature. For surface functionalization of polyimide and polycarbonate, hexamethylenediamine solution $(10 \% \mathrm{v} / \mathrm{v}$ in deionized water) was used with an incubation time of $24 \mathrm{~h}$ at room temperature. For surface functionalization of gold, cysteamine hydrochloride solution $(50 \mathrm{mM}$ in deionized water) was used with an incubation time of $1 \mathrm{~h}$ at room temperature. The surface-functionalized substrates were thoroughly washed with isopropyl alcohol and dried under nitrogen flow before use.

Ex vivo experiments. All porcine tissues and organs used for ex vivo experiments were purchased from a commercial vendor of research-grade porcine tissue (Sierra for Medical Science).

Electrical characterization. To measure electrical impedance, the e-bioadhesive interface was sandwiched between two gold-coated glass electrodes with an overlapped area $(10 \mathrm{~mm} \times 20 \mathrm{~mm})$ and equilibrated in PBS before tests. The gold-coated glass sheets were connected to an impedance analyser $(1287 \mathrm{~A}$, Solartron) to measure the out-of-plane impedance. To measure electrical stability, $0.01 \% \mathrm{w} / \mathrm{v}$ sodium azide was added to the PBS to prevent the growth of microorganisms during the test. To measure electrical properties during deformation, a gold-coated polyester film (McMaster Carr) was cut into a serpentine pattern and adhered to PDMS substrate to minimize fluctuations in interconnect impedance during the measurement.

To measure electrical conductivity, the e-bioadhesive interface was equilibrated in PBS before tests. Based on a modified four-point probe method ${ }^{30}, \mathrm{AC}$ voltage $( \pm 1 \mathrm{~V}, 1,000 \mathrm{~Hz})$ was applied by a power supply (GW Instek), and current $(I)$ and voltage $(V)$ were recorded by a digital multimeter (Keysight $4450 \mathrm{~A})$. The length $(L)$, width $(W)$, and thickness $(T)$ of the sample were measured with a Vernier caliper or a microscope (LV10, Nikon). The electrical conductivity $(\sigma)$ was calculated according to the following equation:

$$
\sigma=\frac{L \times I}{W \times T \times V}
$$

To measure charge injection capacity, a working electrode (the e-bioadhesive interface on platinum with an area of $1 \mathrm{~cm} \times 1 \mathrm{~cm}$ ), a counterelectrode (platinum wire, $0.5 \mathrm{~mm}$ diameter) and a reference electrode $(\mathrm{Ag} / \mathrm{AgCl}$ in saturated $\mathrm{KCl}$ aqueous solution, CHI Instruments) were immersed in PBS. Biphasic pulses of $10 \mathrm{~ms}$ and $\pm 0.5 \mathrm{~V}$ were applied by a multichannel potentiostat (VMP3, Bio-Logic Science Instruments). The output voltage and current were collected to analyse the charge injection capacity.
Mechanical characterization. All samples were adhered by the e-bioadhesive interface by pressing for $5 \mathrm{~s}$ (applying a pressure of $\sim 1 \mathrm{kPa}$ by either a mechanical testing machine or an equivalent weight). The surface of various device materials was functionalized with primary amines before introducing the e-bioadhesive to ensure covalent coupling with the e-bioadhesive interface (Extended Data Fig. 3). All mechanical tests were performed $24 \mathrm{~h}$ after applying the pressure to provide sufficient time for equilibrium swelling of the e-bioadhesive interface in wet environments.

To measure interfacial toughness, adhered samples $2.5 \mathrm{~cm}$ wide were prepared and tested by the standard $180^{\circ}$ peel test (ASTM F2256) or $90^{\circ}$ peel test (ASTM D2861) (for inflexible substrates such as silicon) with a mechanical testing machine (2.5 kN load-cell, Zwick/Roell Z2.5). All tests were conducted with a constant peeling speed of $50 \mathrm{~mm} \mathrm{~min}^{-1}$. Interfacial toughness was calculated by dividing two times the plateau force (for the $180^{\circ}$ peel test) or the plateau force (for the $90^{\circ}$ peel test) by the width of the tissue sample following the corresponding ASTM standard. Poly(methyl methacrylate) films ( $50 \mu \mathrm{m}$ thick, Goodfellow) were applied by using a cyanoacrylate glue (Krazy Glue) as a stiff backing for the tissues. For on-demand detachment, the adhered tissue samples were sprayed with an aqueous triggering solution $(0.5 \mathrm{M}$ sodium bicarbonate and $50 \mathrm{mM} \mathrm{L}$-glutathione reduced in PBS) and kept in a sealed plastic bag for $5 \mathrm{~min}$ before interfacial toughness measurements.

To measure shear strength, the adhered samples with an adhesion area $2.5 \mathrm{~cm}$ wide and $1 \mathrm{~cm}$ long were prepared and tested by the standard lap-shear test (ASTM F2255) with a mechanical testing machine ( $2.5 \mathrm{kN}$ load-cell, Zwick/ Roell Z2.5). All tests were conducted with a constant tensile speed of $50 \mathrm{~mm} \mathrm{~min}^{-1}$. Shear strength was calculated by dividing the maximum force by the adhesion area following the corresponding ASTM standard. Poly(methyl methacrylate) films ( $50 \mu \mathrm{m}$ thick, Goodfellow) were applied by using cyanoacrylate glue as a stiff backing for the tissues.

Validation of amine-coupling of the e-bioadhesive interface. To validate the formation of covalent coupling between primary amine groups and NHS ester groups in the e-bioadhesive interface, the e-bioadhesive interface with and without NHS ester was incubated in PBS with 6-amino fluorescein for $1 \mathrm{~h}$ at room temperature. The incubated samples were then thoroughly washed with deionized water to remove unreacted 6-amino fluorescein. The presence of covalently coupled 6-amino fluorescein was characterized by fluorescence microscopy (LV10, Nikon) (Extended Data Fig. 2).

In vitro biodegradation. In vitro biodegradation evaluation of the e-bioadhesive interface was conducted based on an enzymatic degradation media following the previously reported protocol ${ }^{46}$. To prepare the in vitro enzymatic biodegradation media, $5 \mathrm{mg}$ collagenase was added in $100 \mathrm{ml}$ PBS. Before immersion in the enzymatic media, the samples were sterilized in $70 \%$ ethanol for $15 \mathrm{~min}$ and washed three times with PBS. Each sample was then immersed in $100 \mathrm{ml}$ of the enzymatic media and incubated at $37^{\circ} \mathrm{C}$ with 60 r.p.m. shaking. Sodium azide $(0.01 \% \mathrm{w} / \mathrm{v})$ was added to the enzymatic media to prevent growth of microorganisms during the tests.

In vitro biocompatibility. In vitro biocompatibility tests were conducted using the e-bioadhesive interface-conditioned media as was used for cell culture. To prepare the e-bioadhesive interface-conditioned media, $20 \mathrm{mg}$ of the e-bioadhesive interface was incubated in $1 \mathrm{ml}$ DMEM supplemented with $10 \% \mathrm{v} / \mathrm{v}$ fetal bovine serum and $100 \mathrm{U} \mathrm{ml}^{-1}$ penicillin-streptomycin at $37^{\circ} \mathrm{C}$ for $24 \mathrm{~h}$. Supplemented DMEM without incubating the e-bioadhesive was used as a control. Rat embryonic cardiomyocytes (H9c2(2-1), ATCC) were plated in a confocal dish $(20 \mathrm{~mm}$ diameter) at a density of $0.5 \times 10^{5}$ cells ( $n=3$ per each group). The cells were then treated with the e-bioadhesive interface-conditioned media and incubated at $37^{\circ} \mathrm{C}$ for $24 \mathrm{~h}$ in $5 \% \mathrm{CO}_{2}$. The cell viability was determined by a LIVE/DEAD viability/ cytotoxicity kit for mammalian cells (Thermo Fisher Scientific). A laser confocal microscope (SP 8, Leica) was used to image live cells with excitation/emission at $495 \mathrm{~nm} / 515 \mathrm{~nm}$, and dead cells at $495 \mathrm{~nm} / 635 \mathrm{~nm}$, respectively. The cell viability was calculated by counting the number of live (green fluorescence) and dead (red fluorescence) cells by using ImageJ 1.8.0.

In vivo stability. All animal surgeries were reviewed and approved by the Committee on Animal Care at the Massachusetts Institute of Technology. Female Sprague Dawley rats (225-250 g, Charles River Laboratories) were used for all in vivo studies.

To prepare samples for in vivo subcutaneous implantation, an E-beam evaporator (HHV TF500) was used to deposit gold (100 nm) on the surface of flexible polyimide film ( $25 \mu \mathrm{m}$ thick) to obtain flexible gold-coated polyimide electrodes. Before implantation, the gold-coated polyimide electrodes were functionalized with primary amines according to the method described above. Two gold-coated polyimide electrodes were adhered by the e-bioadhesive interface and cut into rectangular samples $(1 \mathrm{~cm} \times 2 \mathrm{~cm})$. All samples were prepared in an aseptic manner and were further disinfected under ultraviolet light for $3 \mathrm{~h}$.

For implantation in the dorsal subcutaneous space, animals were anaesthetized with isoflurane (1-2\% isoflurane in oxygen) in an anaesthetizing chamber. 
Anaesthesia was maintained using a nose cone. The back hair was removed and the animals were placed in the prone position over a heating pad for the duration of the surgery. The subcutaneous space was accessed by a $1-2 \mathrm{~cm}$ skin incision per implant in the centre of the animal's back. To create space for implant placement, blunt dissection was performed from the incision towards the animal's shoulder blades. The sample was placed in the subcutaneous pocket created above the incision $(n=4)$. The incision was closed with interrupted sutures (4-0 Vicryl, Ethicon) and 3-6 $\mathrm{ml}$ of saline was injected subcutaneously. Fourteen days after the implantation, the animals were killed by $\mathrm{CO}_{2}$ inhalation. The implanted samples were excised and collected for mechanical and electrical measurements. For in vitro comparison, the samples were put in $\mathrm{PBS}$ solution at $37^{\circ} \mathrm{C}$ with 60 r.p.m. shaking for 1 or 14 days.

For measurement of electrical impedance, the two gold-coated polyimide electrodes adhered by the e-bioadhesive interface were directly used according to the method described above. For measurement of interfacial toughness, each end of the two gold-coated polyimide electrodes adhered by the e-bioadhesive interface was gripped by a mechanical testing machine $(2.5 \mathrm{kN}$ load-cell, Zwick/ Roell Z2.5) to perform a $180^{\circ}$ peel test following the method described above. For measurement of electrical conductivity, the e-bioadhesive interface was separated from the gold-coated polyimide electrode with a razor blade and used for four-point probe test according to the method described above.

In vivo biocompatibility. Before implantation, amine-functionalized gold-coated polyimide electrodes with the e-bioadhesive interface were cut into rectangular samples $(1 \mathrm{~cm} \times 1 \mathrm{~cm})$. Gold-coated polyimide electrodes without the e-bioadhesive interface were used as a control group. All samples were prepared in an aseptic manner and were further disinfected under ultraviolet light for $3 \mathrm{~h}$. Implantation in the dorsal subcutaneous space was done as described above. Up to three implants were placed per animal, ensuring no overlap between each subcutaneous pocket created. Fourteen days after the implantation, the animals were killed by $\mathrm{CO}_{2}$ inhalation. Subcutaneous regions of interest were excised and fixed in $10 \%$ formalin for $24 \mathrm{~h}$ for histological analyses $(n=6)$.

In vivo ECG recording. To prepare bioadhesive electrodes, gold foils ( $20 \mu \mathrm{m}$ thick, Goodfellow) were first functionalized with primary amine groups and assembled with the e-bioadhesive interface. The bioadhesive electrodes were then cut into a rectangular strips $(1.3 \mathrm{~mm} \times 2 \mathrm{~mm})$ and a $1-\mathrm{mm}$-diameter sterile biopsy punch (Dynarex) was used to cut a circular hole at the end of the electrode for connection of a lead wire. Two bioadhesive electrodes were assembled with a non-conductive e-bioadhesive interface (based on GO-PVA, $2.5 \mathrm{~mm} \times 12 \mathrm{~mm}$ ). All samples were prepared in an aseptic manner and were further disinfected under ultraviolet light for $3 \mathrm{~h}$.

For implantation of the bioadhesive electrodes, animals were anaesthetized with isoflurane (1-3\% isoflurane in oxygen) in an anaesthetizing chamber. Chest hair was removed. Endotracheal intubation was performed, and the animals were connected to a mechanical ventilator (Model 683, Harvard Apparatus) and placed supine over a heating pad for the duration of the surgery. The heart was exposed via a thoratomy in the third or fourth left intercostal space and the pericardium was removed with fine forceps. To record surface ECG, conventional needle electrodes were used. Anodic and cathodic needle electrodes were inserted into the skin of the right foreleg and the left hindleg of a rat, respectively. To record epicardial ECG, a bioadhesive electrode (cathode) was placed close to the apex of the heart and a second bioadhesive electrode (anode) was placed close to the right atrium $(n=4)$. A needle electrode was inserted into the skin of the left foreleg as a shared ground for both surface and epicardial ECG recordings (Fig. 5a). ECG signals were collected with a Powerlab and a Bio Amplifier (AD Instrument, LabChart Pro 7). Following the ECG recording, the lead wires were removed and the incisions were closed using interrupted sutures (4-0 Vicryl, Ethicon) and $3-6 \mathrm{ml}$ of saline was administered subcutaneously. The animal was ventilated with $100 \%$ oxygen until autonomous breathing was regained, and the intubation catheter was removed.

Fourteen days after implantation each animal was anaesthetized and connected to a ventilator according to the procedure described above. The heart was exposed via a sternotomy and lead wires connected to the bioadhesive electrodes to record epicardial ECG signals according to the procedure described above. Following the ECG recording, a sterile surgical gauze was placed on the surface of bioadhesive electrodes and the triggering solution was applied to the gauze to trigger detachment of the electrodes. Five minutes after applying the triggering solution, the bioadhesive electrodes were gently removed from the heart with forceps. The animals were then killed by $\mathrm{CO}_{2}$ inhalation and the epicardial regions of interest were excised and fixed in $10 \%$ formalin for $24 \mathrm{~h}$ for histological analyses.

In vivo sciatic nerve stimulation. To prepare bioadhesive electrodes for stimulation, gold-coated polyimide films ( $50 \mu \mathrm{m}$ thick) were used according to the procedure described above. The bioadhesive electrodes were cut to $1.5 \mathrm{~mm}$ in width and connected by silver paste to a copper wire. All samples were prepared in an aseptic manner and were further disinfected under ultraviolet light for $3 \mathrm{~h}$. For sciatic nerve stimulation, animals were anaesthetized with isoflurane (1-3\% isoflurane in oxygen) in an anaesthetizing chamber.
After shaving, the vastus lateralis muscle and biceps femoris muscle were dissected to expose the sciatic nerve. The bioadhesive electrodes were adhered on the exposed sciatic nerve. Biphasic charge-balanced rectangular voltage pulses $(1 \mathrm{~Hz}, 0.75-1.2 \mathrm{~V})$ were applied by a function generator (PCSGU250, Velleman). A protractor marker was placed under the leg to measure the change in the angle of the ankle joint.

Histological processing. Fixed tissue samples were placed in $70 \%$ ethanol and submitted for histological processing and haematoxylin and eosin staining at the Hope Babette Tang (1983) Histology Facility in the Koch Institute for Integrative Cancer Research at the Massachusetts Institute of Technology. Histological assessment was performed by a blinded pathologist and representative images of each group are shown in the corresponding figures.

Immunohistochemistry and immunofluoresence analysis. Gold electrodes with and without the e-bioadhesive interface ( $n=3$ per group) were implanted into the dorsal subcutaneous space of the rats as described in the in vivo biocompatibility evaluation. The expression of targeted proteins ( $\alpha$-SMA, CD68, CD3,

collagen-I) was analysed after immunofluorescence and immunohistochemistry staining at 14 days after implantation. Before the immunohistochemistry and immunofluorescence analysis, the paraffin-embedded fixed tissues were sliced and prepared into slides.

For immunohistochemistry analysis, the slides were deparaffinized and rehydrated to deionized water. Antigen retrieval was performed using a steam method during which the slides were steamed in IHC-Tek Epitope Retrieval Solution (IW-1100) for $35 \mathrm{~min}$ and then cooled for $20 \mathrm{~min}$. Then the slides were washed in three changes of PBS for 5 min per cycle and blocked with $3 \% \mathrm{H}_{2} \mathrm{O}_{2}$. After washing in three changes of PBS, the slides were incubated in primary antibodies (1:200 mouse anti- $\alpha$-SMA for fibroblast (ab7817, Abcam); 1:200 mouse anti-CD68 for macrophages (ab201340, Abcam); 1:100 rabbit anti-CD3 for T cells (ab5690, Abcam); 1:200 rabbit anti-collagen-I for collagen (ab21286, Abcam)) diluted with IHC-Tek Antibody Diluent for $1 \mathrm{~h}$ at room temperature. The slides were then washed three times in PBS and incubated with biotinylated goat anti-rabbit or horse anti-mouse secondary antibody (1:500, Vector Lab) for $30 \mathrm{~min}$. The slides were washed in PBS and then incubated with HRP-Streptavidin (1:500, Jackson Immunoresearch) for $30 \mathrm{~min}$. The slides were incubated with DAB Chromogen Substrate Solution (0.05\% 3,3'-diaminobenzidine, IW-1600, IHC World) for 5-10 min and then washed with PBS and counterstained with Mayer's haematoxylin.

For immunofluorescence analysis, the slides were deparaffinized and rehydrated to deionized water. Antigen retrieval was performed using a steam method during which the slides were steamed in IHC-Tek Epitope Retrieval Solution (IW-1100) for $35 \mathrm{~min}$ and then cooled for $20 \mathrm{~min}$. Then the slides were washed in three changes of PBS for 5 min per cycle. After washing, the slides were incubated in primary antibodies (1:200 mouse anti- $\alpha$-SMA for fibroblast (ab7817, Abcam); 1:200 mouse anti-CD68 for macrophages (ab201340, Abcam); 1:100 rabbit anti-CD3 for T cells (ab5690, Abcam); 1:200 rabbit anti-collagen-I for collagen (ab21286, Abcam)) diluted with IHC-Tek Antibody Diluent for $1 \mathrm{~h}$ at room temperature. The slides were then washed three times in PBS and incubated with Alexa Fluor 488 labelled anti-rabbit or anti-mouse secondary antibody (1:200, Jackson Immunoresearch) for $30 \mathrm{~min}$. The slides were washed in PBS and then counterstained with propidium iodide solution for $20 \mathrm{~min}$. A laser confocal microscope (SP 8, Leica) was used for image acquisition. ImageJ was used to quantify the fluorescence intensity of expressed antibodies. All the images were transformed to 8-bit binary images, and the fluorescence intensity was calculated by normalized analysis. All analyses were blinded with respect to the experimental conditions.

Statistical analysis. MATLAB software was used to assess the statistical significance of all comparison studies in this work. Data distribution was assumed to be normal for all parametric tests, but this was not formally tested. In the statistical analysis for comparison between multiple samples, one-way analysis of variance (ANOVA) followed by Tukey's multiple comparison test were conducted with thresholds of ${ }^{\star} P \leq 0.05,{ }^{* *} P \leq 0.01,{ }^{* *} P \leq 0.001$ and ${ }^{* * *} P \leq 0.0001$. For the statistical analysis between two data groups, the two-sample Student's $t$-test was used, and the significance thresholds were ${ }^{\star} P \leq 0.05,{ }^{* *} P \leq 0.01,{ }^{* * *} P \leq 0.001$ and ${ }^{* * * *} P \leq 0.0001$

Reporting Summary. Further information on the research design is available in the Nature Research Reporting Summary linked to this article.

\section{Data availability}

All relevant data that support the findings of this study are available in the article and its supplementary files. Source data of plots are provided with this paper. Source data are provided with this paper.

\section{Code availability}

No custom code is used in this study. 


\section{References}

46. Gorgieva, S. \& Kokol, V. Preparation, characterization, and in vitro enzymatic degradation of chitosan-gelatine hydrogel scaffolds as potential biomaterials. J. Biomed. Mater. Res. A 100, 1655-1667 (2012).

47. Park, S. et al. Self-powered ultra-flexible electronics via nano-grating-patterned organic photovoltaics. Nature 561, 516-521 (2018).

48. Lee, S. et al. Ultrasoft electronics to monitor dynamically pulsing cardiomyocytes. Nat. Nanotechnol. 14, 156-160 (2019).

49. Webb, R. C. et al. Ultrathin conformal devices for precise and continuous thermal characterization of human skin. Nat. Mater. 12, 938-944 (2013).

50. Xu, L. et al. 3D multifunctional integumentary membranes for spatiotemporal cardiac measurements and stimulation across the entire epicardium. Nat. Commun. 5, 3329 (2014).

51. Kim, D.-H. et al. Materials for multifunctional balloon catheters with capabilities in cardiac electrophysiological mapping and ablation therapy. Nat. Mater. 10, 316-323 (2011).

52. Gutruf, P. et al. Wireless, battery-free, fully implantable multimodal and multisite pacemakers for applications in small animal models. Nat. Commun. 10, 5742 (2019).

\section{Acknowledgements}

We thank C.S. Nabzdyk at Mayo Clinic for the insightful discussions and suggestions, R. Bronson at Harvard Medical School for the histological analyses and D. Yang at MIT for help with the charge injection capacity tests. This work is supported by Centers for Mechanical Engineering Research and Education at MIT and SUSTech (MechERE Centers at MIT and SUSTech), the Innovation Committee of Shenzhen Municipality
(JCYJ20170817111714314, C.F.G.), the National Natural Science Foundation of China (numbers U1613204 and 51771089, C.F.G.), the Science Technology the Shenzhen Sci-Tech Fund (KYTDPT20181011104007, C.F.G.) and the Guangdong Innovative and Entrepreneurial Research Team Program (2016ZT06G587, C.F.G.). H.Y. acknowledges financial support from a Samsung Scholarship.

\section{Author contributions}

H.Y., J.D. and X.Z. conceived the idea. J.D., H.Y., C.F.G. and X.Z. designed the study. J.D. and H.Y. developed the materials and methods for the e-bioadhesive interface. J.D., H.Y., J.W. and X.C. designed and performed the in vitro and ex vivo experiments. H.Y., J.W., C.E.V., J.D. and E.T.R. designed the in vivo experiments. J.W., H.Y., C.E.V. and J.D. performed the in vivo experiments. J.D., H.Y., C.F.G. and X.Z. analysed the data and wrote the manuscript with input from all authors.

\section{Competing interests}

The authors declare no competing interests.

\section{Additional information}

Extended data is available for this paper at https://doi.org/10.1038/s41563-020-00814-2. Supplementary information is available for this paper at https://doi.org/10.1038/ s41563-020-00814-2.

Correspondence and requests for materials should be addressed to C.F.G. or X.Z. Reprints and permissions information is available at www.nature.com/reprints. 

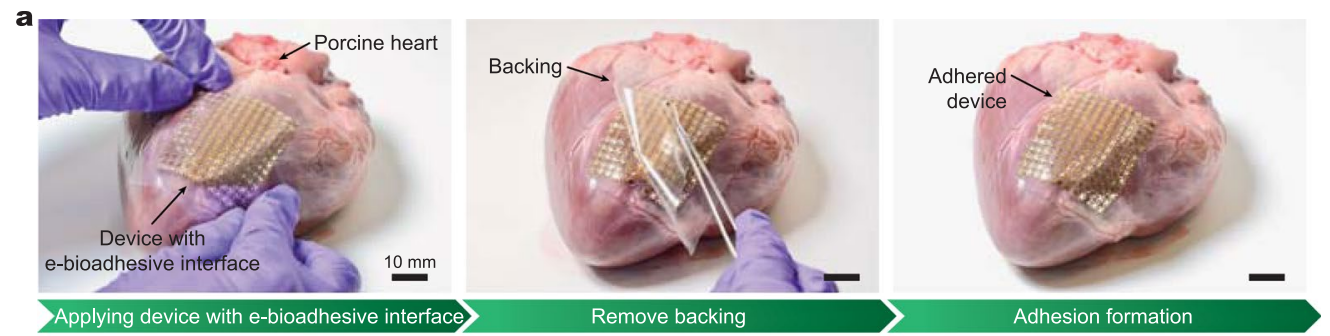

b
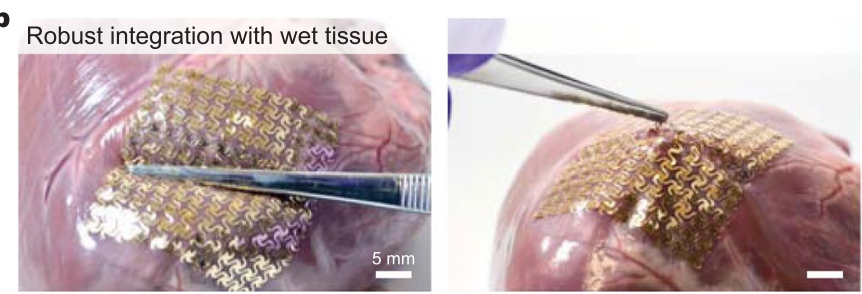

Extended Data Fig. 1 | Rapid and robust adhesion of a device on wet tissue surface by the e-bioadhesive interface. a, Overall process of applying a device with the e-bioadhesive interface to an ex vivo porcine heart. b, Robust integration of the device to the wet heart surface. 
<smiles>CC(C)CC(C)C(=O)ON1C(=O)CCC1=O</smiles>

E-bioadhesive interface with NHS ester

b

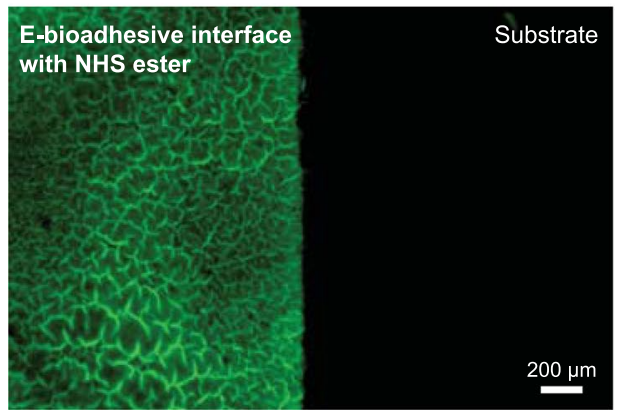

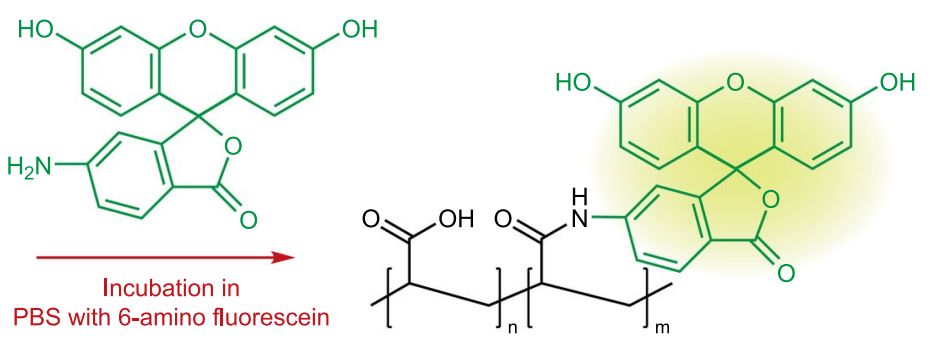

E-bioadhesive interface with fluorescein

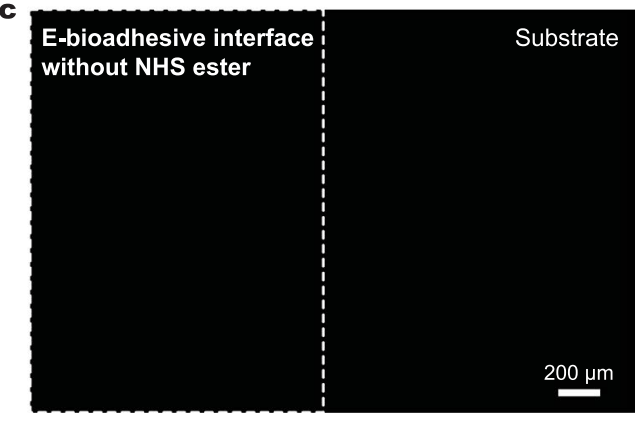

Extended Data Fig. 2 | Amine coupling of the e-bioadhesive interface. a, Schematic illustration of covalent coupling of fluorescein with the e-bioadhesive interface by reaction between NHS ester groups in the e-bioadhesive interface and the primary amine groups in 6-amino fluorescein. $\mathbf{b}$, $\mathbf{c}$, Fluorescence microscopic images of the e-bioadhesive interface with (b) and without (c) NHS ester. 3 independent experiments were conducted with similar results. 
<smiles>CCC[Si](OCC)(OCC)OCC</smiles>

$\stackrel{\begin{array}{l}\text { Oxygen } \\ \text { plasma }\end{array}}{\longrightarrow}$

Silicon, PDMS

b

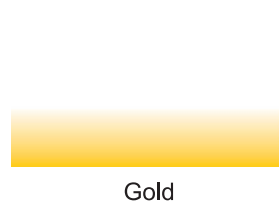

Gold

c
Polyimide
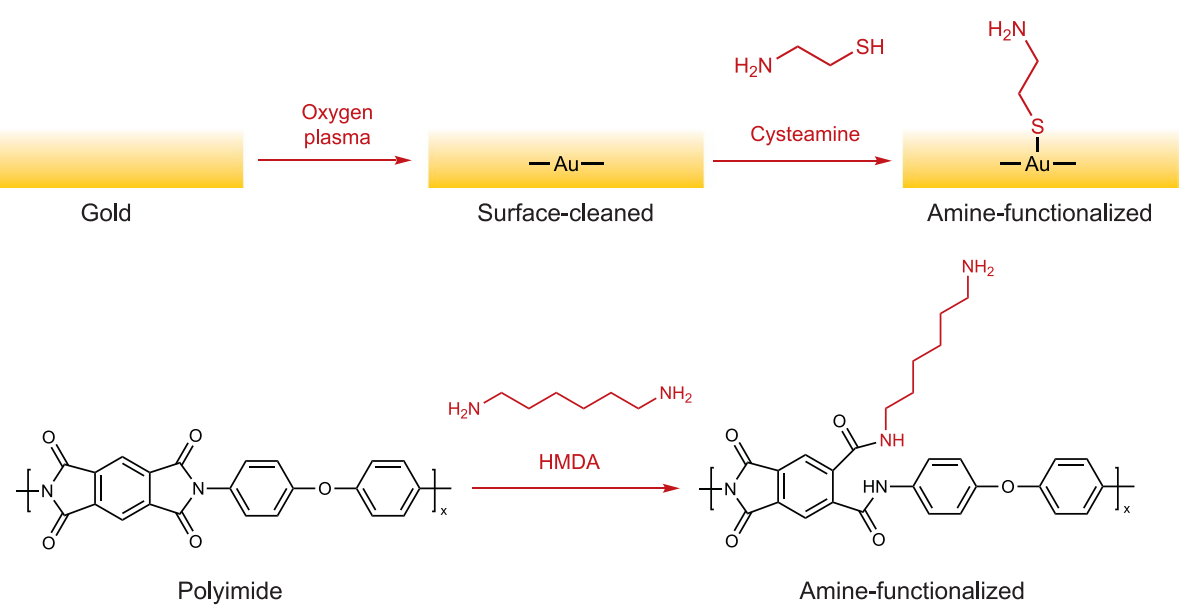

Surface-cleaned

Amine-functionalized

d<smiles>CCCOc1ccc(C(C)(C)c2ccc(OC(C)=O)cc2)cc1</smiles>

Polycarbonate

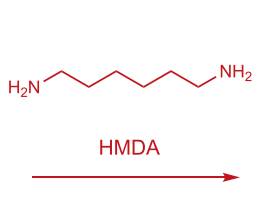

t<smiles>CC(C)(C)Oc1ccc(C(C)(C)c2ccc(OC(C)(C)c3ccc(OC(=O)NCCCCCCN)cc3)cc2)cc1</smiles>

Amine-functionalized

Extended Data Fig. 3 | Surface amine functionalization of various device materials. a, Schematic illustration for primary amine functionalization of silicon and PDMS. b. Schematic illustration for primary amine functionalization of gold. c, Schematic illustration for primary amine functionalization of polyimide. d, Schematic illustration for primary amine functionalization of polycarbonate. 


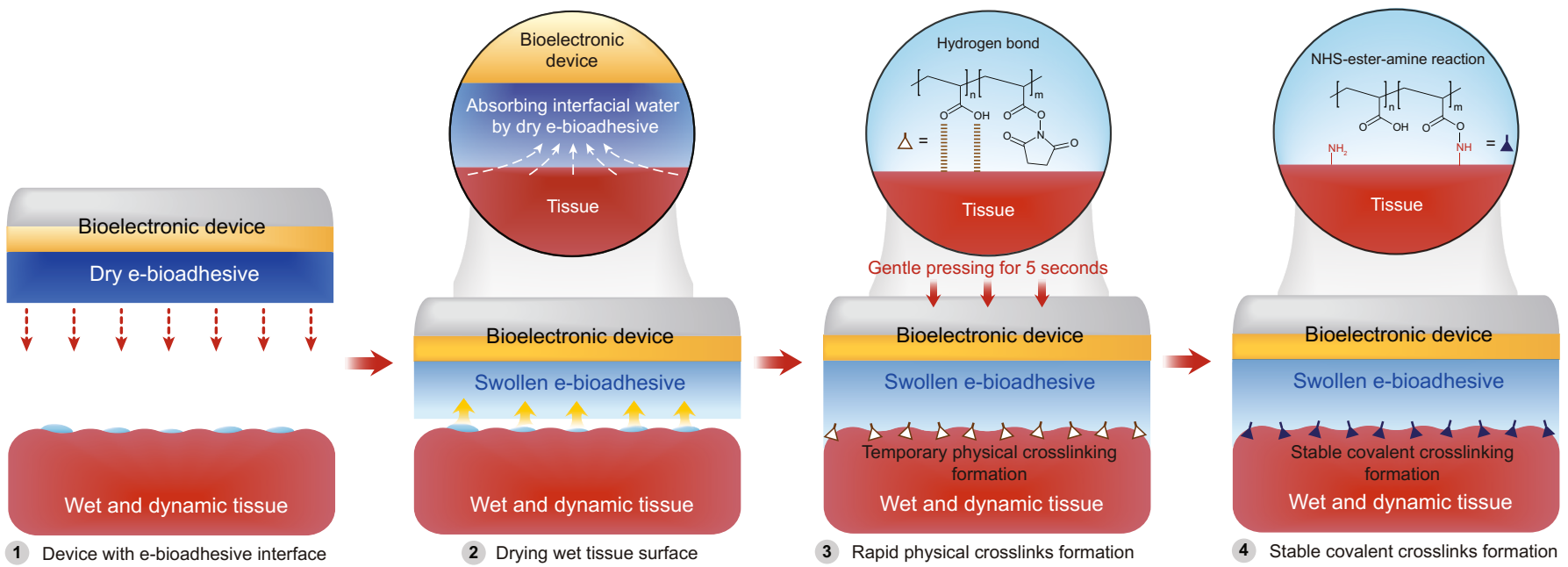

Extended Data Fig. 4 | Schematic illustrations for the overall application process and mechanism of the e-bioadhesive interface. The e-bioadhesive interface is assembled with bioelectronic devices to endow the ability to adhere to and electrically communicate with wet tissue surfaces. Upon contact with the wet tissue surface, the e-bioadhesive interface quickly absorbs the interfacial water and dry the tissue surface. The carboxylic acid groups in the e-bioadhesive interface form temporary physical crosslinks by hydrogen bonds and/or electrostatic interactions. The NHS ester groups in the e-bioadhesive interface subsequently form covalent crosslinks with the primary amine groups on the tissue surface and provide stable long-term tissue-device integration. 


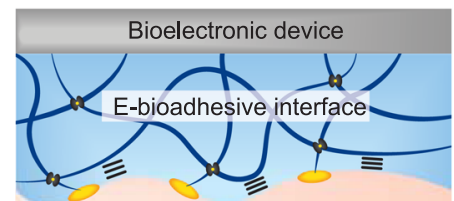

Triggering solution application
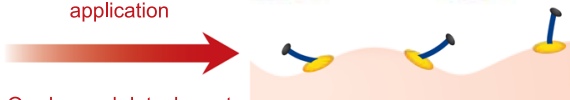

Tissue

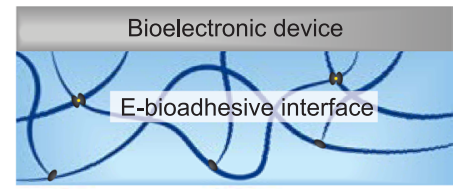

I

On-demand detachment

Tissue

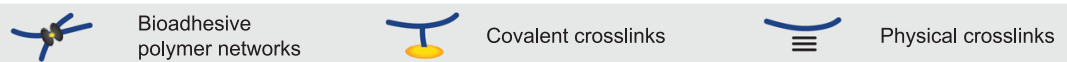

b

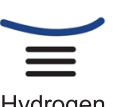

Hydrogen bond
1 Covalent crosslinks

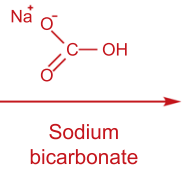

Tissue

bicarbonate<smiles>CC(C)(C)CC(C)(C)C(=O)[O-]</smiles>

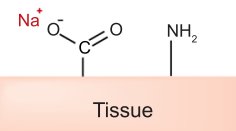

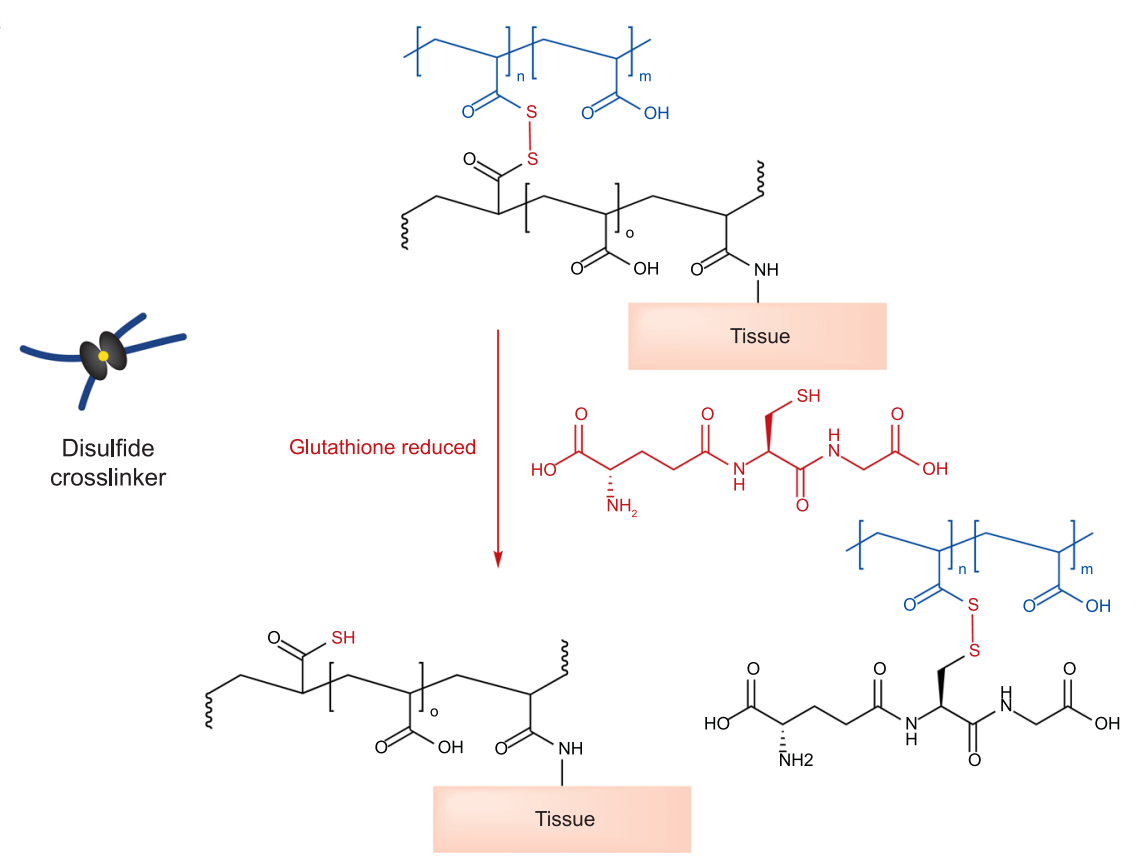

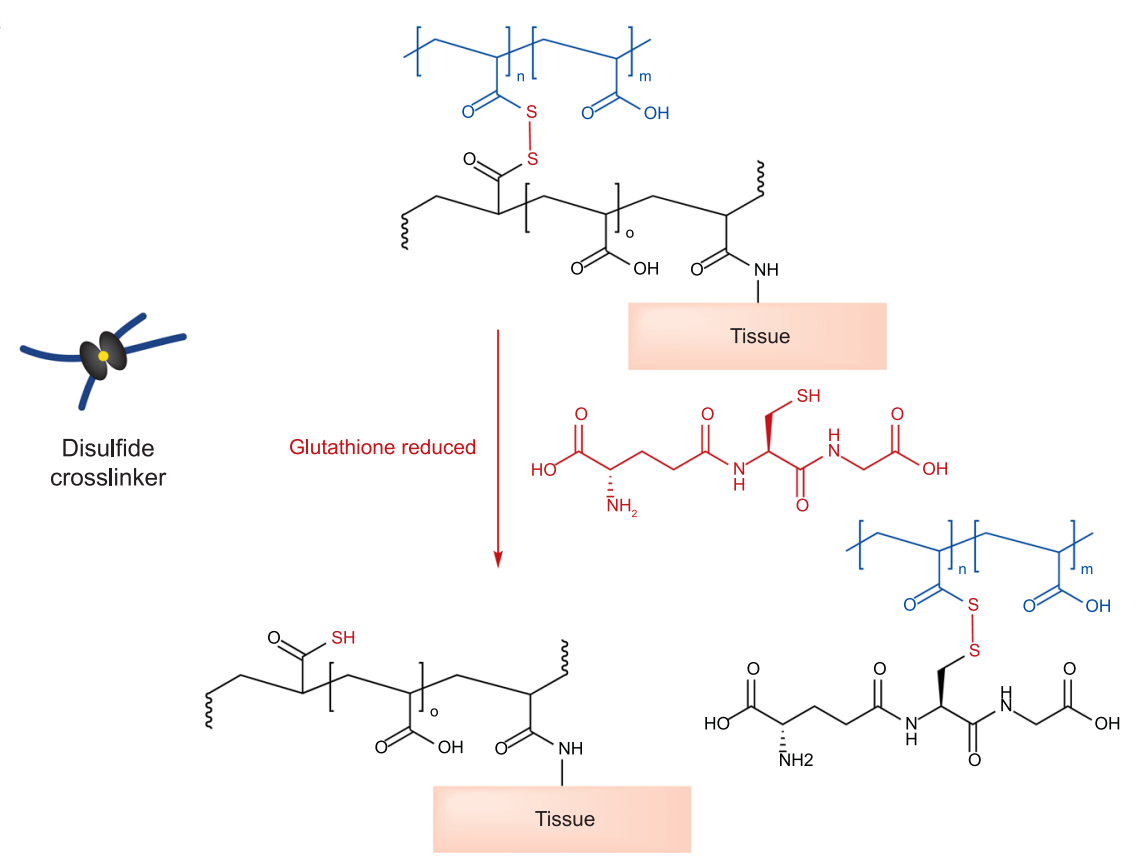

Disulfide crosslinker

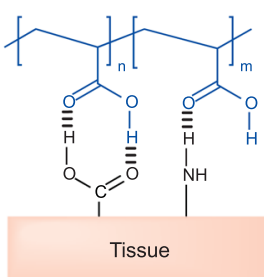

$$
\text { in }
$$

Extended Data Fig. 5 | On-demand detachment of the e-bioadhesive interface. a, Schematic illustrations for on-demand detachment of the e-bioadhesive interface by the triggering solution. $\mathbf{b}$, Schematic illustrations for chemistry of the on-demand cleaving of hydrogen bonds between the e-bioadhesive interface and the tissue surface. $\mathbf{c}$, Schematic illustrations for the on-demand cleaving of covalent disulfide bonds between the e-bioadhesive interface and the tissue surface. 
a

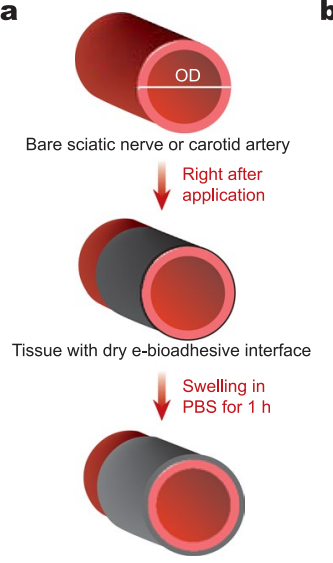

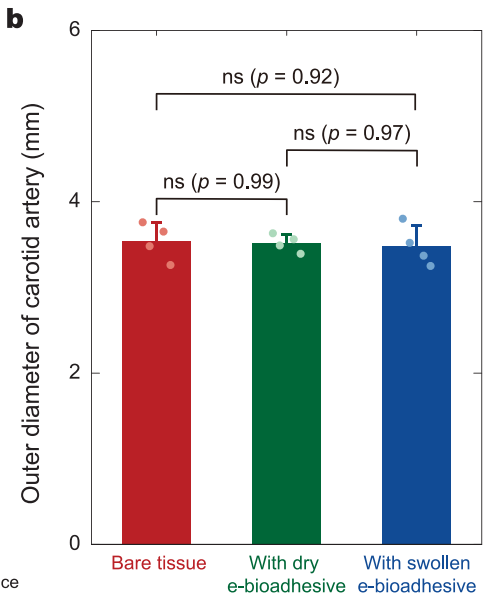

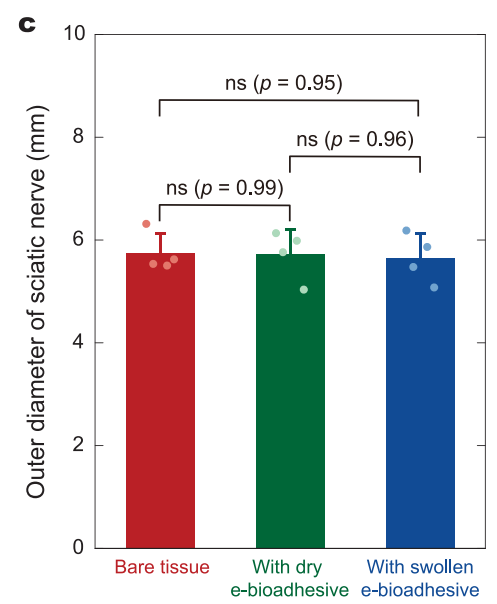

Extended Data Fig. 6 | Evaluation of compressive narrowing of carotid artery and sciatic nerve by the e-bioadhesive interface. a, Schematic illustrations of a measurement setup for the potential compressive narrowing of tissues by the e-bioadhesive interface. $\mathbf{b}$, Outer diameter of bare carotid artery, carotid artery with the dry and fully swollen e-bioadhesive interfaces. c, Outer diameter of bare sciatic nerve, sciatic nerve with the dry and fully swollen e-bioadhesive interfaces. Values in $\mathbf{b}, \mathbf{c}$ represent the mean and the standard deviation ( $n=4$ independent samples). Statistical significance and $P$ values are determined by two-sided Student $t$-test; ns, not significant. 
a

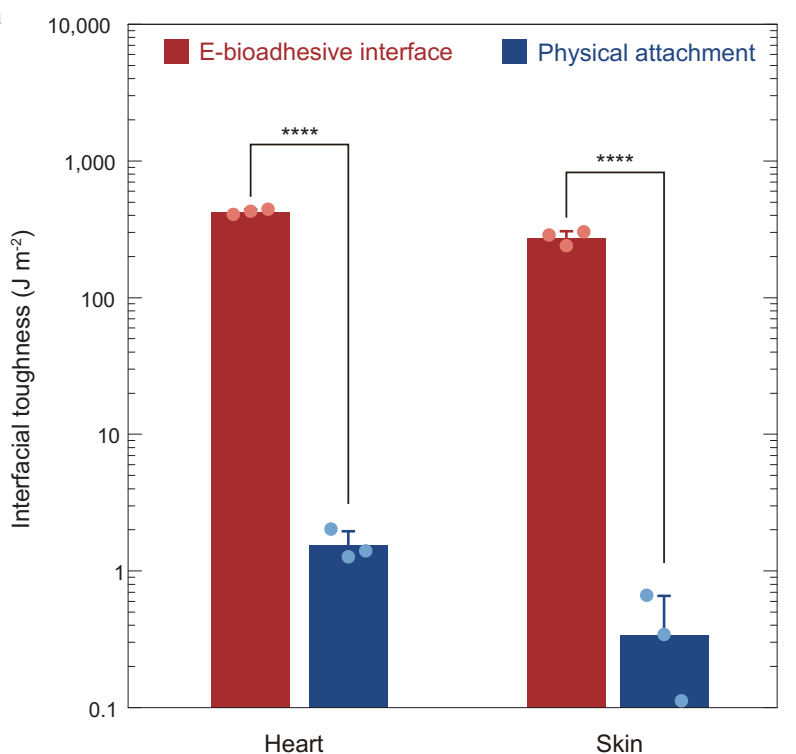

b

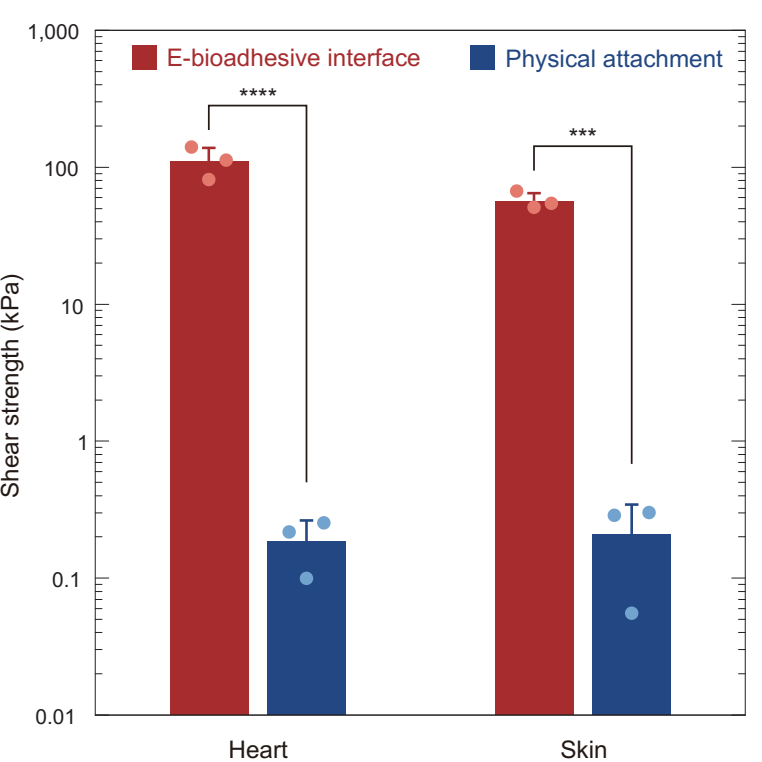

Extended Data Fig. 7 | Adhesion performance of device integration to wet tissues by physical attachment. a, Interfacial toughness between various tissues and polyimide substrates adhered by the e-bioadhesive interface and physical attachment. $\mathbf{b}$, Shear strength between various tissues and polyimide substrates adhered by the e-bioadhesive interface and physical attachment. Values in $\mathbf{a}, \mathbf{b}$ represent the mean and the standard deviation $\left(n=3\right.$ independent samples). Statistical significance and $P$ values are determined by two-sided Student t-test; ${ }^{\star \star \star} p \leq 0.001 ;{ }^{\star \star \star \star} p \leq 0.0001$. 


\section{NATURE MATERIALS}

ARTICLES
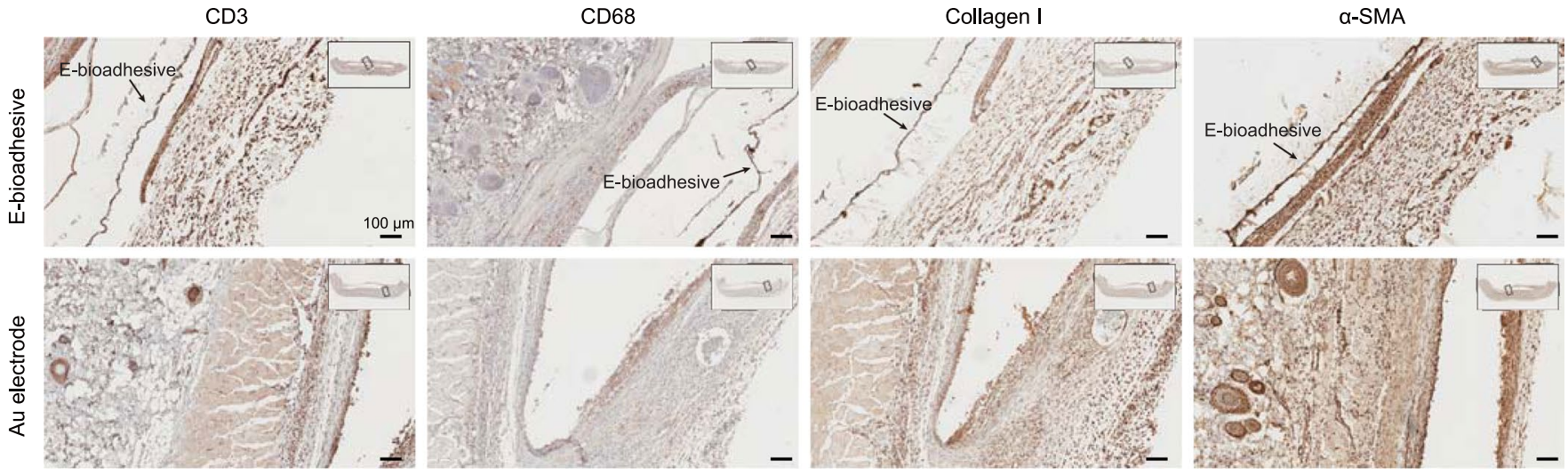

Extended Data Fig. 8 | Immunohistochemistry analysis of CD3, CD68, Collagen-I, and $\alpha$-SMA expression. Representative immunohistochemistry images of CD3, CD68, Collagen-I, and $\alpha$-SMA for gold electrode with and without the e-bioadhesive interface after rat subcutaneous implantation for 14 days, respectively. 5 independent experiments were conducted with similar results. 

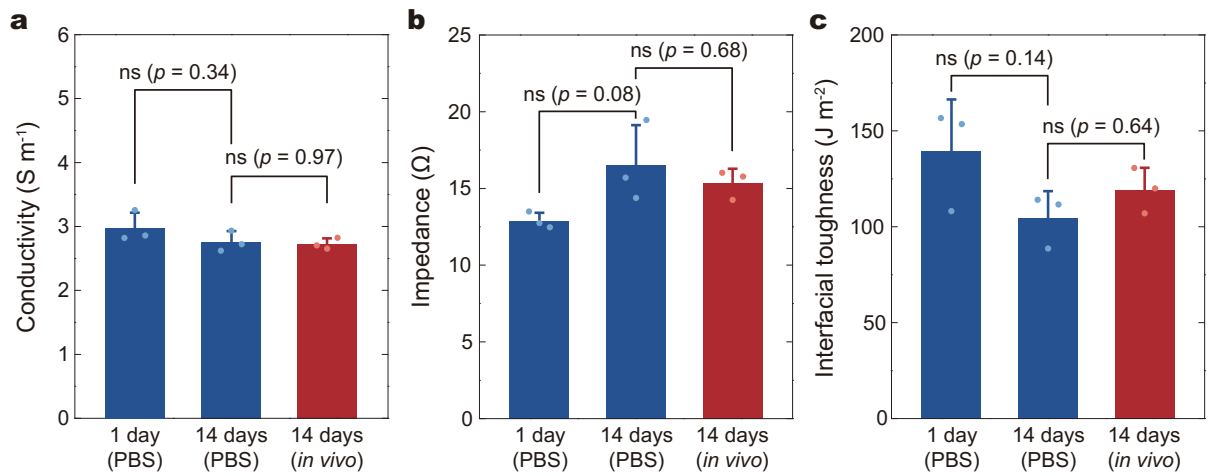

Extended Data Fig. 9 | Stability of in vivo performance. Conductivity, impedance, and interfacial toughness of two gold electrodes adhered by the e-bioadhesive interface after 1 day and 14 days of incubation in PBS, and 14 days of in vivo implantation in rat dorsal subcutaneous pockets. Values represent the mean and the standard deviation ( $n=3$ independent samples). Statistical significance and $P$ values are determined by two-sided Student t-test; ns, not significant. 
NATURE MATERIALS

ARTICLES

Extended Data Table 1 | Comparison of various types of bioelectronic devices for wet tissues and organs

\begin{tabular}{|c|c|c|c|c|c|c|}
\hline Type & Device Thickness & $\begin{array}{l}\text { Integration } \\
\text { Mechanism }\end{array}$ & $\begin{array}{l}\text { Adhesion } \\
\text { Energy }\end{array}$ & $\begin{array}{l}\text { Long-term } \\
\text { Stability }\end{array}$ & Tissue Damage & Examples \\
\hline Imperceptible Electronics & $<3 \mu \mathrm{m}$ & Physical attachment & $\begin{array}{c}\text { Low } \\
\left(<3 \mathrm{~J} \mathrm{~m}^{-2}\right)\end{array}$ & $\begin{array}{l}\text { Low } \\
\text { (acute function) }\end{array}$ & $\begin{array}{c}\text { Low } \\
\text { (surface contact) }\end{array}$ & $(12,47,48)$ \\
\hline Conformable Electronics & $5-30 \mu \mathrm{m}$ & Physical attachment & $\begin{array}{c}\text { Low } \\
\left(<3 \mathrm{~J} \mathrm{~m}^{-2}\right)\end{array}$ & $\begin{array}{l}\text { Low } \\
\text { (acute function) }\end{array}$ & $\begin{array}{c}\text { Low } \\
\text { (surface contact) }\end{array}$ & $(7,9,11,44,49,50)$ \\
\hline Flexible Electronics & $>30 \mu \mathrm{m}$ & Suture & $\begin{array}{c}\text { High } \\
\left(>500 \mathrm{~J} \mathrm{~m}^{-2}\right)\end{array}$ & $\begin{array}{c}\text { High } \\
\text { (>6 days, heart) }\end{array}$ & $\begin{array}{c}\text { High } \\
\text { (tissue puncture) }\end{array}$ & $(15,16,19,51,52)$ \\
\hline E-bioadhesive Interface & $\begin{array}{l}\text { Thickness } \\
\text { independent }\end{array}$ & $\begin{array}{l}\text { Dry-crosslinking } \\
\text { mechanism }\end{array}$ & $\begin{array}{c}\text { High } \\
\left(>450 \mathrm{~J} \mathrm{~m}^{-2}\right)\end{array}$ & $\begin{array}{c}\text { High } \\
\text { (> 14 days, heart) }\end{array}$ & $\begin{array}{c}\text { Low } \\
\text { (surface contact) }\end{array}$ & This work \\
\hline
\end{tabular}




\section{natureresearch}

Corresponding author(s): Xuanhe Zhao

Last updated by author(s): 08/20/2020

\section{Reporting Summary}

Nature Research wishes to improve the reproducibility of the work that we publish. This form provides structure for consistency and transparency in reporting. For further information on Nature Research policies, see Authors \& Referees and the Editorial Policy Checklist.

\section{Statistics}

For all statistical analyses, confirm that the following items are present in the figure legend, table legend, main text, or Methods section.

n/a Confirmed

$\bigotimes$ The exact sample size $(n)$ for each experimental group/condition, given as a discrete number and unit of measurement

$\square$ \ A statement on whether measurements were taken from distinct samples or whether the same sample was measured repeatedly

The statistical test(s) used AND whether they are one- or two-sided

Only common tests should be described solely by name; describe more complex techniques in the Methods section.

$\bigotimes$ A description of all covariates tested

$\square$ \A description of any assumptions or corrections, such as tests of normality and adjustment for multiple comparisons

$\triangle$ A full description of the statistical parameters including central tendency (e.g. means) or other basic estimates (e.g. regression coefficient)

AND variation (e.g. standard deviation) or associated estimates of uncertainty (e.g. confidence intervals)

For null hypothesis testing, the test statistic (e.g. $F, t, r$ ) with confidence intervals, effect sizes, degrees of freedom and $P$ value noted

Give $P$ values as exact values whenever suitable.

Х $\square$ For Bayesian analysis, information on the choice of priors and Markov chain Monte Carlo settings

Х $\square$ For hierarchical and complex designs, identification of the appropriate level for tests and full reporting of outcomes

$\bigotimes \square$ Estimates of effect sizes (e.g. Cohen's $d$, Pearson's $r$ ), indicating how they were calculated

Our web collection on statistics for biologists contains articles on many of the points above.

\section{Software and code}

Policy information about availability of computer code

Data collection Electrocardiogram (ECG) was collected by LabChart Pro 7 (ADInstruments).

Data analysis Microscopic images were analyzed by using ImageJ (Version: 2.1.0). All statistical analyses were performed by using MATLAB (Version: R2018b, MathWorks).

For manuscripts utilizing custom algorithms or software that are central to the research but not yet described in published literature, software must be made available to editors/reviewers. We strongly encourage code deposition in a community repository (e.g. GitHub). See the Nature Research guidelines for submitting code \& software for further information.

\section{Data}

Policy information about availability of data

All manuscripts must include a data availability statement. This statement should provide the following information, where applicable:

- Accession codes, unique identifiers, or web links for publicly available datasets

- A list of figures that have associated raw data

- A description of any restrictions on data availability

All data is available in the main text or the supplementary information.

\section{Field-specific reporting}

Please select the one below that is the best fit for your research. If you are not sure, read the appropriate sections before making your selection. 


\section{Life sciences study design}

All studies must disclose on these points even when the disclosure is negative.

Sample size In vivo experiments on rat were conducted to investigate in vivo biocompatibility based on histological assessment after implantation, in vivo ECG recording, and in vivo sciatic nerve stimulation. The appropriate sample sizes $(n=4-5)$ were selected based on the published literatures on ECG recording and sciatic nerve stimulation.

Data exclusions The following rats were also excluded: Animals that did not survive the surgery, animals that showed infection or opened the sutured incision, and animals with defective samples.

Replication In vivo studies for biocompatibility were reliably reproduced based on comparable histological assessment for each case by the blinded pathologist. In vivo studies for ECG recording and sciatic nerve stimulation were reliably reproduced based on comparable recorded signals for different animals. All in vivo studies were independently performed with at least 1 day between surgeries.

Randomization All the tests were performed with randomly allocated experimental groups.

Blinding All histological assessments were conducted by the blinded pathologist based on randomly mixed histological slides without informing type or study group of samples.

\section{Reporting for specific materials, systems and methods}

We require information from authors about some types of materials, experimental systems and methods used in many studies. Here, indicate whether each material, system or method listed is relevant to your study. If you are not sure if a list item applies to your research, read the appropriate section before selecting a response.

\begin{tabular}{l|l} 
Materials \& experimental systems \\
\hline$n / a$ & Involved in the study \\
\hline & $\bigotimes$ Antibodies \\
$\square$ & $\bigotimes$ Eukaryotic cell lines \\
$\square$ & $\square$ Palaeontology \\
$\square$ & $\square$ Animals and other organisms \\
$\square$ & $\square$ Clinical data
\end{tabular}

\begin{tabular}{l|l}
\multicolumn{2}{l}{ Methods } \\
\hline n/a & Involved in the study \\
$\searrow$ & $\square$ ChIP-seq \\
$\searrow$ & $\square$ Flow cytometry \\
$\searrow$ & $\square$ MRI-based neuroimaging
\end{tabular}

\section{Antibodies}

Antibodies used

Primary antibodies: Mouse anti-aSMA for fibroblast (ab7817, Abcam); Rabbit anti-Collagen I for Collagen (ab21286, Abcam);

Mouse anti-CD68 for macrophages (ab201340, Abcam); Rabbit anti-CD 3 for T-cells (ab5690, Abcam).

Secondary antibodies: Alexa Fluor 488 labeled anti-rabbit or anti-mouse secondary antibody (315-545-003, Jackson Immunoresearch).

Validation
All antibodies are commercially available and have been tested by the manufacturer. Vendors and catalog numbers are listed above and validation can be found there.
Mouse anti-aSMA (ab7817, Abcam): This monoclonal antibody recognizes aSMA. Manufacturer-validated to react with Mouse, Rat, Rabbit, Human, Pig aSMA (https://www.abcam.com/alpha-smooth-muscle-actin-antibody-1a4-ab7817.html).
Rabbit anti-Collagen I (ab21286, Abcam): This polyclonal antibody recognizes to Collagen I. Manufacturer-validated to react with Mouse, Rat Collagen I (https://www.abcam.com/collagen-i-antibody-ab21286.html).
Mouse anti-CD68 (ab201340, Abcam): This monoclonal antibody recognizes CD68. Manufacturer-validated to react with Mouse, Rat, Human CD68 (https://www.abcam.com/cd68-antibody-c68684-ab201340.html).
Rabbit anti-CD3 (ab5690, Abcam): This polyclonal antibody recognizes to CD3. Manufacturer-validated to react with Mouse, Rat, Human CD3 (https://www.abcam.com/cd3-antibody-ab5690.html).

\section{Eukaryotic cell lines}

Policy information about cell lines

Cell line source(s)

Rat embryonic cardiomyocytes (H9c2(2-1), ATCC) 
Mycoplasma contamination

Commonly misidentified lines (See ICLAC register)
The cell line was not tested for mycoplasma contamination.

No commonly misidentified cell lines were used

\section{Animals and other organisms}

Policy information about studies involving animals; ARRIVE guidelines recommended for reporting animal research

Laboratory animals

Wild animals

Field-collected samples

Ethics oversight
Female Sprague Dawley rats (12 weeks, 225-275g weight), were purchased from Charles River Laboratories.

This study does not involve wild animals.

This study does not involve field-collected samples.

Animal procedures were reviewed and approved by the Massachusetts Institute of Technology Committee on Animal Care.

Note that full information on the approval of the study protocol must also be provided in the manuscript. 